\title{
INVARIANCE D'UN CONVEXE FERMÉ PAR UN SEMI-GROUPE ASSOCIÉ À UNE FORME NON-LINÉAIRE
}

\author{
LOUISE BARTHÉLEMY
}

\begin{abstract}
The invariance of a closed and convex set under a semigroup $S(t)$ associated with a nonlinear form is investigated. Other properties of increase and domination of the semigroup $S(t)$ are also derived. Examples are also given to demonstrate the power of the theoretical results.
\end{abstract}

\section{INTRODUCTION}

Soit $H$ un espace de Hilbert sur $\mathbb{K}=\mathbb{R}$ ou $\mathbb{C}$. Etant donnés $V$ un autre espace de Hilbert sur $\mathbb{K}$ s'injectant continûment dans $H$ avec image dense, et $a(u, v)$ une forme sesquilinéaire sur $V \times V$ continue, positive et elliptique (c'est-à-dire $\operatorname{Re} a(u, u) \geq 0$, et $\operatorname{Re} a(u, u)+\|u\|_{H}^{2} \geq c\|u\|_{V}^{2}$ pour $u \in V$, avec $c>0$ ), on associe à $(V, a)$ un semi-groupe $S(t)$ continu (en fait analytique), de contractions (linéaires) sur $H$ dont le générateur infinitésimal est la restriction à $H$ de $u \rightarrow a(u, \cdot)$.

Récemment E. M. Ouhabaz dans [8] a caractérisé à l'aide de $a(u, v)$ l'invariance par $S(t)$ d'un convexe fermé $C$ de $H$ et, dans le cas où $H$ est réticulé il caractérise la positivité de $S(t)$, la domination de $S(t)$ par un autre semi-groupe $T(t)$ associé à un couple $(W, b)$, et il applique, entre autres, ses résultats à des formes différentielles du type,

$$
a(u, v)=\sum_{i, j} \int_{\Omega} a_{i j} D_{i} u D_{j} v d x+\sum_{i} \int_{\Omega}\left(a_{i} u D_{i} v+b_{i} v D_{i} u\right) d x+\int_{\Omega} b u v d x
$$

sur $V \times V$, avec $V$ un sous espace fermé de $H^{1}(\Omega), \Omega$ un ouvert de $\mathbb{R}^{\mathbb{N}}$, $H=L^{2}(\Omega), a_{i j}, a_{i}, b_{i}, b \in L^{\infty}(\Omega)$ avec

$$
\sum_{i, j} a_{i j} \xi_{i} \xi_{j} \geq c|\xi|^{2} \text { p.p. pour tout } \xi \in \mathbb{R}^{\mathbb{N}} \text { où } c>0 .
$$

1991 Mathematics Subject Classification. Primary 47H20;

Key words and phrases. Hilbert space, invariance of closed and convex sets, semigroups associated with nonlinear forms.

Received: November 21, 1995. 
Dans cet article on étend ses résultats au cas non linéaire. Pour cela on se donne $V$ un espace de Banach réel tel que $V$ s'injecte continûment dans $H, a(u, v)$ une forme sur $V \times V$ hémicontinue en $u$, linéaire continue en $v$, monotone dans le sens $a(u, u-\hat{u}) \geq a(\hat{u}, u-\hat{u})$ et $\Phi: V \rightarrow]-\infty, \infty]$ convexe semi-continue inférieurement telle que le domaine effectif $D(\Phi)=$ $\{v \in V ; \Phi(v)<\infty\}$ est non vide. Moyennant une hypothèse de coercivité (cf. (1.6)) on peut associer à $(V, a, \Phi)$ un semi-groupe continu de contractions (non linéaires) sur $\overline{D(\Phi)}$ (adhérence de $D(\Phi)$ dans $H$ ). On note $S(t)$ la composition de ce semi-groupe avec la projection sur le convexe $\overline{D(\Phi)}$. Par abus de langage on dira encore que $S(t)$ est le semi-groupe sur $H$ associé à $(V, a, \Phi)$ (on a $S(t+s)=S(t) \circ S(s), t \rightarrow S(t) u$ est continue, $S(t)$ est une contraction de $H$, mais $S(0) \neq I d_{H}$ si $D(\Phi)$ n'est pas dense dans $\left.H\right)$.

Dans une première partie on montre que si $C$ est un convexe fermé de $H$ et si $P_{C}$ est la projection sur $C$ alors,

$S(t) C \subset C$ si et seulement si pour tout $u \in D(\Phi), P_{C} u \in D(\Phi)$ et

$$
\Phi\left(P_{C} u\right) \leq \Phi(u)+a\left(u, u-P_{C} u\right) .
$$

Dans la deuxième partie on prend $H=L^{2}(\Omega, \mathcal{B}, \mu ; \mathbb{R})$ où $(\Omega, \mathcal{B}, \mu)$ est un espace mesuré, on caractérise la croissance de $S(t)$ sur $H$, ainsi que la comparaison de $S(t)$ avec $T(t)$ semi-groupe sur $H$ associé à un autre triplet $(W, b, \Psi)$. Plus précisément si $S(t)$ et $T(t)$ laissent invariant un convexe fermé $\Gamma$ et si $S(t)$ ou $T(t)$ est croissant sur $\Gamma$, on caractérise

$$
S(t) u \leq T(t) u \text { pour tout } u \in \Gamma \text { et tout } t \geq 0 .
$$

Dans la troisième partie on prend $H=L^{2}(\Omega, \mathcal{B}, \mu ; \mathbb{K})$ avec $\mathbb{K}=\mathbb{R}$ ou $\mathbb{C}$ et étant donnés $S(t)$ et $T(t)$ des semi-groupes sur $H$ associés à des triplets $(V, a, \Phi)$ et $(W, b, \Psi)$ respectivement, si l'on suppose $T(t) \geq 0$ et croissant sur $H_{\mathbb{R}}^{+}=L^{2}\left(\Omega, \mathcal{B}, \mu ; \mathbb{R}^{+}\right)$on caractérise la domination de $S(t)$ par $T(t)$ c'est-àdire

$$
|S(t) u| \leq T(t)|u| \quad \text { pour tout } u \in H \text { et tout } t \geq 0 .
$$

Dans les sections 4 et 5 on traite deux exemples d'applications à des formes différentielles concrètes.

Dans la partie $4, H=L^{2}(] 0,1[), V=W^{1, p}(] 0,1[),<p<\infty$,

$$
\begin{aligned}
& a(u, v)=\int_{0}^{1}\left[a_{0}\left(\cdot, u, u^{\prime}\right) v+a_{1}\left(\cdot, u, u^{\prime}\right) v^{\prime}\right] d x, \\
& \Phi(u)=\varphi(u(0), u(1))
\end{aligned}
$$

et l'on caractérise la croissante de $S(t)$ à l'aide de $\varphi$.

Dans la partie $5, H=L^{2}(\Omega)$ où $\Omega$ est un ouvert borné régulier de $\mathbb{R}^{\mathbb{N}}$,

$$
\begin{aligned}
& V=W^{1, p}(\Omega) \cap L^{2}(\Omega), 1<p<\infty, \\
& a(u, v)=\int_{\Omega} a_{0}(\cdot, D u) \cdot D v d x, \\
& \Phi(u)=\int_{\bar{\Omega}} \varphi(\cdot, u) d \mu \text { où } \mu \text { est une mesure de Radon positive sur } \bar{\Omega}
\end{aligned}
$$

d'énergie finie relativement à $V$ et $\varphi$ une intégrande convexe normale sur $\bar{\Omega} \times \mathbb{R}$; 
l'on donne des conditions nécessaires et des conditions suffisantes de comparaison et de domination de $S(t)$ et de $T(t)$ (semi-groupe associé à un autre triplet $V, b, \Psi)$ du même type que $(V, a, \Phi)$.

L'étude du cas non linéaire apporte un éclaircissement nouveau sur le cas linéaire.

\section{RÉSUltats GÉNÉRAuX D'INVARIANCE}

Soit $H$ est un espace de Hilbert réel de produit scalaire $(\cdot, \cdot)$ et de norme $\|\cdot\|$. Pour tout sous-ensemble $E$ de $H$ on notera $\bar{E}$ la fermeture de $E$ dans $H$ et, pour tout convexe fermé $C$ de $H, P_{C}$ désignera la projection de $H$ sur $C$.

On se donne $V$ un sous-espace vectoriel de $H, a$ une application de $V \times V$ dans $\mathbb{R}$ et $\Phi$ une application de $V$ dans ] $-\infty,+\infty$ ] vérifiant les hypothèses suivantes:

(1.1) $V$ est un espace de Banach réflexif de norme $\|\cdot\|_{V} \geq\|\cdot\|$, de dual $V^{\prime}$;

(1.2) $a(u,.) \in V^{\prime}$ pour tout $u \in V$;

(1.3) $a(u, \hat{u}-u) \leq a(\hat{u}, \hat{u}-u)$ pour tout $u, \hat{u} \in V$ (monotonie);

(1.4) $\lim _{t \downarrow 0} a(u+t v, w)=a(u, w)$ pour tout $u, v, w \in V$ (hémicontinuité);

(1.5) $\Phi$ est convexe, semi-continue inférieurement (s.c.i) de domaine effectif $D(\Phi)=\{u \in V ; \Phi(u)<+\infty\}$ non vide;

(1.6) pour tout $u_{0} \in D(\Phi), \lim _{\|u\|_{V} \rightarrow+\infty} \frac{a\left(u, u-u_{0}\right)+\Phi(u)+\|u\|^{2}}{\|u\|_{V}}=+\infty$ (coercivité).

On note $A_{a, \Phi}$ l'opérateur de $H$ associé à $a$ et $\Phi$ défini par,

$$
v \in A_{a, \Phi}(u) \Longleftrightarrow\left\{\begin{array}{l}
u \in D(\Phi), v \in H \text { et, pour tout } w \in V \\
\Phi(u)+(v, w-u) \leq \Phi(w)+a(u, w-u)
\end{array}\right.
$$

$A_{a, \Phi}$ est un opérateur maximal monotone de $H$ (cf. Remarque 1.6), $-A_{a, \Phi}$ engendre donc un semi-groupe continu de contractions sur $\overline{D\left(A_{a, \Phi}\right)}$ convexe fermé de $H$, noté $e^{-t A_{a, \Phi}}$ pour $t \geq 0$ (cf. [2]).

Rappelons que pour $u_{0} \in D\left(A_{a, \Phi}\right) \quad u(t)=e^{-t A_{a, \Phi}} u_{0}$ est l'unique fonction de $\left[0, \infty\left[\rightarrow H\right.\right.$ localement absolument continue vérifiant $u^{\prime}(t)+A_{a, \Phi} u(t) \ni$ $0 \quad p p t \geq 0, u(0)=u_{0}$.

Notons alors pour tout $t \geq 0$

$$
S_{a, \Phi}(t)=e^{-t A_{a, \Phi}} \circ P_{\overline{D\left(A_{a, \Phi}\right)}}
$$

Par abus de langage, on dira que $S_{a, \Phi}(t)$ est le semi-groupe sur $H^{1}$ associé $\grave{a}(V, a, \Phi)$.

Enfin si $C$ est un convexe fermé de $H$ on notera $I_{C}: V \rightarrow[0, \infty]$, l'application convexe s.c.i définie par $I_{C}(u)=0$ si $u \in C \cap V$ et $I_{C}(u)=+\infty$ sinon. On peut alors énoncer le résultat principal de ce papier,

\footnotetext{
${ }^{1}$ on a: pour tout $t \geq 0 S_{a, \Phi}(t)$ est une contraction de $H$ dans $H, S_{a, \Phi}(t+s)=$ $S_{a, \Phi}(t) \circ S_{a, \Phi}(s)$ pour tout $t, s \in[0, \infty], t \rightarrow S_{a, \Phi}(t) u$ est continu sur $[0, \infty[$ pour tout $u \in H$, mais $S_{a, \Phi}(0) \neq I d_{H}$ si $D\left(A_{a, \Phi}\right)$ n'est pas dense dans $H$.
} 
Théorème 1.1. Soit $C$ un convexe fermé de $H$. On note $S(t)=S_{a, \Phi}(t)$ et $S_{C}(t)=S_{a, \Phi+I_{C}}(t)$.

Les assertions suivantes sont équivalentes:

i) Pour tout $u \in C$ et tout $t \geq 0, S(t) u \in C$;

ii) Pour tout $u \in D(\Phi), P_{C} u \in D(\Phi)$ et

$$
\Phi\left(P_{C} u\right) \leq \Phi(u)+a\left(P_{C} u, u-P_{C} u\right) ;
$$

iii) Pour tout $u \in D(\Phi), P_{C} u \in D(\Phi)$ et

$$
\Phi\left(P_{C} u\right) \leq \Phi(u)+a\left(u, u-P_{C} u\right)
$$

iv) Pour tout $u \in C$ et tout $t \geq 0$

$$
S(t) u=S_{C}(t) u .
$$

Exemple 1.2. Si $\Phi=0$ et $C$ est un sous-espace vectoriel fermé de $H$, alors, $S(t) u \in C$ pour tout $u \in C$ et tout $t \geq 0$, si et seulement si $P_{C} u \in V$ pour tout $u \in V$ et $a(u, v)=0$ pour tout $u \in V \cap C$ et tout $v \in V \cap C^{\perp}$.

Exemple 1.3. Soit $H=L^{2}(\Omega, \mathcal{B}, \mu ; \mathbb{R})$ où $(\Omega, \mathcal{B}, \mu)$ est un espace mesuré ; $H$ est muni du produit scalaire usuel $(u, v)=\int u v d \mu$.

Alors les assertions suivantes sont équivalentes:

i) Pour tout $u \geq 0$ et tout $t \geq 0, S(t) u \geq 0$;

$$
\begin{aligned}
& \text { ii) Pour tout } u \in D(\Phi), u^{+} \in D(\Phi) \text { et } \Phi\left(u^{+}\right)+a\left(u^{+}, u^{-}\right) \leq \Phi(u) \text {; } \\
& \text { iii) Pour tout } u \in D(\Phi), u^{+} \in D(\Phi) \text { et } \Phi\left(u^{+}\right)+a\left(u, u^{-}\right) \leq \Phi(u) \text {. }
\end{aligned}
$$

Lorsque ces propriétés sont vérifiées on dit que $S(t)$ est positif.

Exemple 1.4. Dans cet exemple on montre comment le théorème 1.1 étend les résultats de E. M. Ouhabaz ([7], [8]).

$H_{\mathbb{C}}$ est un espace de Hilbert complexe de produit scalaire $(\cdot, \cdot)_{\mathbb{C}}$ et de norme $\|\|,. a_{\mathbb{C}}: D\left(a_{\mathbb{C}}\right) \times D\left(a_{\mathbb{C}}\right) \rightarrow \mathbb{C}$ est une forme sesquilinéaire de domaine $D\left(a_{\mathbb{C}}\right), \mathbb{C}$-sous-espace vectoriel de $H_{\mathbb{C}}$. On suppose que $a_{\mathbb{C}}$ est positive, continue et fermée c'est-à-dire, vérifie,

$$
\left\{\begin{array}{cl}
i) \quad \operatorname{Re} a_{\mathbb{C}}(u, u) \geq 0 \quad \text { pour tout } \quad u \in D\left(a_{\mathbb{C}}\right) ; \\
i i) \quad a_{\mathbb{C}} \text { est continue pour la norme }\|\cdot\|_{a_{\mathbb{C}}} \text { de } D\left(a_{\mathbb{C}}\right) \text { définie par } \\
\|u\|_{a_{\mathbb{C}}}^{2}=\operatorname{Re} a_{\mathbb{C}}(u, u)+\|u\|^{2} ; \\
\text { iii }) \quad\left(D\left(a_{\mathbb{C}}\right),\|\cdot\|_{a_{\mathbb{C}}}\right) \quad \text { est un espace de Hilbert. }
\end{array}\right.
$$

Soit $A$ l'opérateur $\mathbb{C}$-linéaire (multivoque) de $H_{\mathbb{C}}$ défini par $v \in A u \Longleftrightarrow u \in D\left(a_{\mathbb{C}}\right), v \in H_{\mathbb{C}}$ et $(v, w)_{\mathbb{C}}=a_{\mathbb{C}}(u, w)$ pour tout $w \in D\left(a_{\mathbb{C}}\right)$.

Si $D\left(a_{\mathbb{C}}\right)$ est dense dans $H_{\mathbb{C}} A$ est univoque et il est connu (cf. par exemple [6]) que $-A$ engendre un semi-groupe holomorphe $e^{-t A}$ sur $H_{\mathbb{C}}$.

D'autre part si l'on prend pour $H, H_{\mathbb{C}}$ muni du produit scalaire réel $(\cdot, \cdot)=$ $\operatorname{Re}(\cdot, \cdot)_{\mathbb{C}}$, il est clair que le triplet $\left(V=D\left(a_{\mathbb{C}}\right), a=R e a_{\mathbb{C}}, \Phi=0\right)$ vérifie 
les hypothèses (1.1) à (1.6). Alors l'opérateur $A_{a_{\mathbb{C}}}$ défini par (1.7) avec $a=\operatorname{Re} a_{\mathbb{C}}$ et $\Phi=0$ coïncide avec $A$. En effet,

$$
v \in A_{a_{\mathbb{C}}} \Longleftrightarrow u \in D\left(a_{\mathbb{C}}\right), v \in H_{\mathbb{C}} \text { et } \operatorname{Re}(v, w)_{\mathbb{C}}=\operatorname{Re}\left(a_{\mathbb{C}}(u, w)\right)
$$

pour tout $w \in D\left(a_{\mathbb{C}}\right)$, et l'on remarque que:

$\operatorname{Re}(v, w)_{\mathbb{C}}=\operatorname{Re} a_{\mathbb{C}}(u, w)$ pour tout $w \in D\left(a_{\mathbb{C}}\right) \Longrightarrow \operatorname{Im}(v, w)_{\mathbb{C}}=\operatorname{Re}(v, i w)_{\mathbb{C}}$ $=\operatorname{Re} a_{\mathbb{C}}(u, i w)=\operatorname{Im} a_{\mathbb{C}}(u, w)$ pour tout $w \in D\left(a_{\mathbb{C}}\right)$.

Donc, si $D\left(a_{\mathbb{C}}\right)$ est dense dans $H_{\mathbb{C}}, S_{a_{\mathbb{C}}}(t)$ défini par $(1.8)$ associé à $A_{a_{\mathbb{C}}}$ coïncide avec $e^{-t A}$. Dans le cas où $D\left(a_{\mathbb{C}}\right)$ n'est pas dense, $S_{a_{\mathbb{C}}}(t)$ coïncide avec le semi-groupe considéré par E. M. Oubahaz dans [8].

Supposons de plus que $H_{\mathbb{C}}=H_{\mathbb{R}} \oplus i H_{\mathbb{R}}$ où $H_{\mathbb{R}}$ est un $\mathbb{R}$-sous-espace vectoriel fermé de $H_{\mathbb{C}}$ vérifiant

$$
(u, v)_{\mathbb{C}} \in \mathbb{R} \quad \text { pour tout } \quad u, v \in H_{\mathbb{R}} .
$$

Pour $u \in H_{\mathbb{C}}$ on note $\operatorname{Re} u$ (resp. Im $u$ ) la projection de $u$ (resp. -iu) sur $H_{\mathbb{R}}$ parallèlement à $i H_{\mathbb{R}}$, on a alors $u=R e u+i \operatorname{Im} u$. L'hypothèse (1.14) implique que $R e u=P_{H_{\mathbb{R}}} u$. Alors d'après l'exemple 1.2. on peut affirmer que les assertions suivantes sont équivalentes:

$$
\left\{\begin{array}{r}
\text { i) Pour tout } u \in H_{\mathbb{R}} \quad \text { et tout } t \geq 0, S_{a_{\mathbb{C}}}(t) u \in H_{\mathbb{R}} ; \\
\text { ii) Pour tout } u \in D\left(a_{\mathbb{C}}\right), \operatorname{Re} u \in D\left(a_{\mathbb{C}}\right) \text { et } \\
\text { pour tout } u, v \in D\left(a_{\mathbb{C}}\right) \cap H_{\mathbb{R}}, a_{\mathbb{C}}(u, v) \in \mathbb{R} .
\end{array}\right.
$$

Lorsque ces propriétés sont vérifiées on dit que $S_{a_{\mathbb{C}}}(t)$ est réel.

Si $H_{\mathbb{C}}=L^{2}(\Omega, \mathcal{B}, \mu ; \mathbb{C})$ muni du produit scalaire $(u, v)_{\mathbb{C}}=\int u \bar{v} d \mu, H_{\mathbb{R}}=$ $L^{2}(\Omega, \mathcal{B}, \mu ; \mathbb{R}), H_{\mathbb{R}}^{+}=L^{2}\left(\Omega, \mathcal{B}, \mu ; \mathbb{R}^{+}\right)$avec $(\Omega, \mathcal{B}, \mu)$ un espace mesuré, alors la linéarité de $S_{a_{\mathbb{C}}}(t),(1.12)$ et (1.15) nous permettent d'affirmer que:

1) $S_{a_{\mathbb{C}}}(t) H_{\mathbb{R}}^{+} \subset H_{\mathbb{R}}^{+}$pour tout $t \geq 0 \Longrightarrow S_{a_{\mathbb{C}}}(t) H_{\mathbb{R}} \subset H_{\mathbb{R}}$ pour tout $t \geq 0$;

2) Si $S_{a_{\mathbb{C}}}(t) H_{\mathbb{R}} \subset H_{\mathbb{R}}$ pour tout $t \geq 0$, alors les propriétés suivantes sont équivalentes:

i) Pour tout $u \in H_{\mathbb{R}}^{+}$et tout $t \geq 0, S_{a_{\mathbb{C}}}(t) u \in H_{\mathbb{R}}^{+}$;

ii) Pour tout $u \in D\left(a_{\mathbb{C}}\right) \cap H_{\mathbb{R}}, u^{+} \in D\left(a_{\mathbb{C}}\right)$ et $a_{\mathbb{C}}\left(u^{+}, u^{-}\right) \leq 0$;

iii) Pour tout $u \in D\left(a_{\mathbb{C}}\right) \cap H_{\mathbb{R}}, u^{+} \in D\left(a_{\mathbb{C}}\right)$ et $a_{\mathbb{C}}\left(u, u^{-}\right) \leq 0$.

Exemple 1.5. a) Soit $H=L^{2}(\Omega, \mathcal{B}, \mu ; \mathbb{K})$ où $(\Omega, \mathcal{B}, \mu)$ est un espace mesuré, $\mathbb{K}=\mathbb{R}$ ou $\mathbb{C}, H$ est muni du produit scalaire réel $(u, v)=\operatorname{Re} \int u \bar{v} d \mu$. Pour $u \in H$ on note: $\|u\|_{\infty}=\sup _{x \in \Omega} e s s|u(x)|$, sign $u$ défini pour presque tout $x \in \Omega$ par sign $u(x)=\frac{u(x)}{|u(x)|}$ si $u(x) \neq 0$, sign $u(x)=0$ si $u(x)=0$, et si

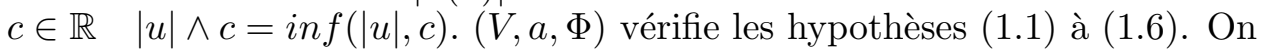
pose $S(t)=S_{a, \Phi}(t)$. Alors les assertions suivantes sont équivalentes:

i) Pour tout $u \in H$ et tout $t \geq 0,\|S(t) u\|_{\infty} \leq\|u\|_{\infty}$; 
ii) Pour tout $c \in] 0, \infty[$ et tout $u \in D(\Phi),(|u| \wedge c)$ sign $u \in D(\Phi)$ et

$$
\left.\Phi(|u| \wedge c) \operatorname{sign} u) \leq \Phi(u)+a(|u| \wedge c) \operatorname{sign} u,(|u|-c)^{+} \operatorname{sign} u\right) ;
$$

iii) Pour tout $c \in] 0, \infty[$ et tout $u \in D(\Phi),(|u| \wedge c)$ sign $u \in D(\Phi)$ et

$$
\Phi((|u| \wedge c) \text { signu }) \leq \Phi(u)+a\left(u,(|u|-c)^{+} \text {signu }\right) .
$$

En effet, si pour $c \in] 0, \infty\left[\right.$ l'on désigne par $B_{c}$ le convexe fermé de $H$ défini par

alors

$$
B_{c}:=\{u \in H ;|u| \leq c\}
$$

i) $\Longleftrightarrow$ pour tout $c \in] 0, \infty\left[\right.$, pour tout $u \in B_{c}$ et tout $t \geq 0, \quad S(t) u \in B_{c}$.

Il suffit donc d'appliquer le théorème 1.1 en remarquant que,

$$
P_{B_{c}} u=(|u| \wedge c) \operatorname{sign} u \quad \text { et } u-P_{B_{c}} u=(|u|-c)^{+} \text {signu. }
$$

b) Reprenons l'exemple linéaire 1.4 avec $H_{\mathbb{C}}=L^{2}(\Omega, \mathcal{B}, \mu ; \mathbb{C}) . S_{a_{\mathbb{C}}}(t)$ étant linéaire, la propriété i) précédente est équivalente à , pour tout $u \in B_{1}$ et $t \geq 0 \quad S_{a_{\mathbb{C}}}(t) u \in B_{1}$. Alors en traduisant le résultat de a) on retrouve la caractérisation de E. M. Ouhabaz de la $L^{\infty}$ contractivité:

les assertions suivantes sont équivalentes:

i) Pour tout $u \in H$ et tout $t \geq 0,\left\|S_{a_{\mathbb{C}}}(t) u\right\|_{\infty} \leq\|u\|_{\infty}$;

ii) Pour tout $u \in D\left(a_{\mathbb{C}}\right),(|u|-1)^{+}$signu $\in D\left(a_{\mathbb{C}}\right)$ et $\operatorname{Re}_{\mathbb{C}}((|u| \wedge 1)$ signu, $(|u|-1)^{+}$signu $) \geq 0$

iii) Pour tout $u \in D\left(a_{\mathbb{C}}\right),(|u|-1)^{+}$sign $u \in D\left(a_{\mathbb{C}}\right)$ et $\operatorname{Rea}_{\mathbb{C}}(u,(|u|-$ $\left.1)^{+} \operatorname{sign} u\right) \geq 0$.

Avant de démontrer le théorème faisons quelques remarques concernant l'opérateur $A=A_{a, \Phi}$.

Remarque 1.6. Il est classique que lorsque $V$ est dense dans $H, A$ est maximal monotone dans $H$ (cf. [3]). Or nous ne faisons pas cette hypothèse; voyons comment s'y ramener. Notons $H_{0}=\bar{V}$ espace de Hilbert s'injectant continûment dans $V^{\prime}$ et $H_{0}^{\perp}$ son orthogonal dans $H$.

Alors, comme on vient de le rappeler, l'opérateur $A_{0}$ de $H_{0}$ défini par

$$
v \in A_{0} u \Longleftrightarrow\left\{\begin{array}{l}
u \in D(\Phi), v \in H_{0} \text { et pour tout } w \in V \\
\Phi(u)+(v, w-u) \leq \Phi(w)+a(u, w-u)
\end{array}\right.
$$

est maximal monotone dans $H_{0}$. On en déduit la maximale monotonie de $A$ en remarquant que $D(A)=D\left(A_{0}\right)$ et pour $u \in D(A) \quad A u=A_{0} u+H_{0}^{\perp}$.

Remarque 1.7. Si $A$ est un opérateur maximal monotone de $H$ et si $C$ est un convexe fermé de $H$ on sait (cf. [2], Proposition 4.5) que

$$
S(t) C \subset C \text { pour tout } t \geq 0 \Longleftrightarrow(I+\lambda A)^{-1} C \subset C \text { pour tout } \lambda>0,
$$

où $S(t)=e^{-t A} o P_{\overline{D(A)}}$ et $I$ est l'identité de $H$.

Le lemme suivant est fondamental pour démontrer le théorème 1.1. 
Lemme 1.8. Soit $A=A_{a, \Phi}$ et pour $\lambda>0 \quad J_{\lambda}=(I+\lambda A)^{-1}$ sont les résolvantes de A. Alors,

1) $\overline{D(A)}=\overline{D(\Phi)}=\overline{D(\partial \Phi)}$

2) Si $u \in D(\Phi)$ alors lorsque $\lambda \downarrow 0, J_{\lambda} u \rightarrow u$ dans $H, J_{\lambda} u \rightarrow u$ pour $\sigma\left(V, V^{\prime}\right), a\left(J_{\lambda} u, w\right) \rightarrow a(u, w)$ pour tout $w \in V, a\left(J_{\lambda} u, J_{\lambda} u\right) \rightarrow$ $a(u, u)$ et $\Phi\left(J_{\lambda} u\right) \rightarrow \Phi(u)$.

Preuve du lemme. 1) Comme $D(A) \subset D(\partial \Phi) \subset D(\Phi), \quad \overline{D(A)}=\overline{D(\Phi)}=$ $\overline{D(\partial \Phi)} \Longleftrightarrow D(\Phi) \subset \overline{D(A)}$.

Soit $u \in D(\Phi)$; notons $u_{\lambda}=J_{\lambda} u=(I+\lambda A)^{-1} u$. Comme $\frac{u-u_{\lambda}}{\lambda} \in A u_{\lambda}$ on a d'après (1.7) et (1.3), pour tout $w \in D(\Phi)$,

$$
\begin{aligned}
\Phi\left(u_{\lambda}\right)+\frac{1}{\lambda}\left(u-u_{\lambda}, w-u_{\lambda}\right) & \leq \Phi(w)+a\left(u_{\lambda}, w-u_{\lambda}\right) \\
& \leq \Phi(w)+a\left(w, w-u_{\lambda}\right) .
\end{aligned}
$$

Appliquons (1.16) avec $w=u$, il vient,

$$
\begin{aligned}
a\left(u, u_{\lambda}-u\right)+\Phi\left(u_{\lambda}\right) & \leq a\left(u_{\lambda}, u_{\lambda}-u\right)+\Phi\left(u_{\lambda}\right) \\
& \leq \frac{-1}{\lambda}\left\|u-u_{\lambda}\right\|^{2}+\Phi(u) \leq \Phi(u)
\end{aligned}
$$

d'où

$$
\frac{a\left(u_{\lambda}, u_{\lambda}-u\right)+\Phi\left(u_{\lambda}\right)+\left\|u_{\lambda}\right\|^{2}}{\left\|u_{\lambda}\right\|_{V}} \leq \frac{\Phi(u)+\left\|u_{\lambda}\right\|^{2}}{\left\|u_{\lambda}\right\|_{V}} \leq \frac{\Phi(u)}{\left\|u_{\lambda}\right\|_{V}}+\left\|u_{\lambda}\right\| .
$$

Or on sait que $u_{\lambda} \rightarrow \underline{u}=P_{\overline{D(A)}} u$ dans $H$ (cf. [2]), on en déduit d'après (1.6) que $\left(u_{\lambda}\right)_{\lambda>0}$ est bornée dans $V$.

Maintenant on montre que $\underline{u}=u$ ce qui implique $u \in \overline{D(A)}$.

On sait (cf. [9] théorème 3.18) que $D(\partial \Phi)$ est dense dans $D(\Phi)$ pour la topologie de $V$, donc il existe $\left(\alpha_{n}, \beta_{n}\right) \in \partial \Phi$ telle que $\alpha_{n} \rightarrow u$ dans $V$. Utilisant (1.16) avec $w=\alpha_{n}$ il vient que,

$$
\begin{aligned}
\left(u-u_{\lambda}, \alpha_{n}-u_{\lambda}\right) & \leq \lambda\left(\Phi\left(\alpha_{n}\right)-\Phi\left(u_{\lambda}\right)+a\left(\alpha_{n}, \alpha_{n}-u_{\lambda}\right)\right) \\
& \leq \lambda\left(<\beta_{n}, \alpha_{n}-u_{\lambda}>_{V^{\prime}, V}+a\left(\alpha_{n}, \alpha_{n}-u_{\lambda}\right)\right) .
\end{aligned}
$$

$\left(u_{\lambda}\right)_{\lambda>0}$ étant bornée dans $V$ et $u_{\lambda} \rightarrow \underline{u}$ dans $H$, lorsque $\lambda \downarrow 0$, on a $(u-$ $\left.\underline{u}, \alpha_{n}-\underline{u}\right) \leq 0$, puis faisant $n \rightarrow \infty$ il vient que $\|u-\underline{u}\|^{2} \leq 0$ d'où $u=\underline{u}$.

2) On a donc $u_{\lambda} \rightarrow u$ dans $H$ et $\left(u_{\lambda}\right)_{\lambda>0}$ bornée dans $V$, on en déduit que $u_{\lambda} \rightarrow u$ pour $\sigma\left(V, V^{\prime}\right)$. $\Phi$ étant s.c.i. on tire alors de (1.17) que $\Phi\left(u_{\lambda}\right) \rightarrow \Phi(u)$ et $a\left(u_{\lambda}, u_{\lambda}-u\right) \rightarrow 0$ lorsque $\lambda \downarrow 0$.

Enfin utilisant (1.3) il est aisé de vérifier que pour tout $t>0$ et tout $w \in V$

$$
\liminf _{\lambda \downarrow 0} a\left(u_{\lambda}, w\right) \geq a(u-t w, w),
$$

puis utilisant (1.4), il vient que $a\left(u_{\lambda}, w\right) \rightarrow a(u, w)$ et $a\left(u_{\lambda}, u_{\lambda}\right) \rightarrow a(u, u)$ lorsque $\lambda \downarrow 0$.

Preuve du théorème 1.1. On note $A=A_{a, \Phi}, S(t)=S_{a, \Phi}(t)$ et pour $\lambda>$ $0 J_{\lambda}=(I+\lambda A)^{-1}$. 
1) On montre que $i) \Longrightarrow i i)$ : en faisant $t=0$ dans i) on a d'après le lemme 1.8

$$
P_{\overline{D(\Phi)}} u=P \overline{\overline{D(A)}} u \in C \quad \text { pour tout } u \in C
$$

ce qui est équivalent à,

$$
P_{C} u \in \overline{D(\Phi)} \text { pour tout } u \in D(\Phi) .^{2}
$$

Maintenant soient $u \in D(\Phi)$ et $v=P_{C} u \in \overline{D(\Phi)}$. Pour $\lambda>0$, on a d'après (1.16) avec $v, J_{\lambda} v, u$ au lieu de $u, u_{\lambda}, w$ respectivement,

$$
\begin{aligned}
a\left(J_{\lambda} v, J_{\lambda} v-u\right)+\Phi( & \left.J_{\lambda} v\right) \leq \Phi(u)+\frac{1}{\lambda}\left(v-J_{\lambda} v, J_{\lambda} v-u\right) \\
= & \Phi(u)-\frac{1}{\lambda}\left\|v-J_{\lambda} v\right\|^{2}+\frac{1}{\lambda}\left(v-J_{\lambda} v, v-u\right) \\
\leq &
\end{aligned}
$$

car $v=P_{C} u$ et $J_{\lambda} v \in C$ d'après la remarque 1.7 ; on a donc

$$
\frac{a\left(J_{\lambda} v, J_{\lambda} v-u\right)+\Phi\left(J_{\lambda} v\right)+\left\|J_{\lambda} v\right\|^{2}}{\left\|J_{\lambda} v\right\|_{V}} \leq \frac{\Phi(u)}{\left\|J_{\lambda} v\right\|_{V}}+\left\|J_{\lambda} v\right\| .
$$

$\left(J_{\lambda} v\right)_{\lambda>0}$ étant bornée dans $H$, d'après (1.6) on en déduit que $\left(J_{\lambda} v\right)_{\lambda>0}$ est bornée dans $V$. Comme $v \in \overline{D(A)}=\overline{D(\Phi)}, J_{\lambda} v \rightarrow v$ dans $H$ et $J_{\lambda} v \rightarrow v$ pour $\sigma\left(V, V^{\prime}\right)$ lorsque $\lambda \downarrow 0$. D'après (1.18) et (1.3) on a alors $\Phi(v) \leq$ $\liminf \Phi\left(J_{\lambda} v\right) \leq \Phi(u)+a(u, u-v)$ donc $v \in D(\Phi)$. D'après le lemme 1.8 et (1.18) on en déduit la relation (1.9) du théorème.

2) Montrons iii $\Rightarrow$ iv): Tout d'abord il est clair que $\Phi+I_{C}$ est convexe s.c.i sur $V$ et que $\Phi+I_{C} \geq \Phi$ entraine que $\left(a, \Phi+I_{C}\right)$ vérifie l'hypothèse de coercivité (1.6) donc $S_{C}(t)=S_{a, \Phi+I_{C}}(t)$ est défini. Soit $u \in C$. Utilisons (1.10) avec $u_{\lambda}=J_{\lambda} u \in D(\Phi)$ et (1.16) avec $u, u_{\lambda}$ et $w=P_{C} u_{\lambda} \in D(\Phi)$, il vient que,

$$
\begin{aligned}
0 & \leq \Phi\left(u_{\lambda}\right)-\Phi\left(P_{C} u_{\lambda}\right)+a\left(u_{\lambda}, u_{\lambda}-P_{C} u_{\lambda}\right) \\
& \leq \frac{1}{\lambda}\left(u-u_{\lambda}, u_{\lambda}-P_{C} u_{\lambda}\right) \\
& =\frac{1}{\lambda}\left(u-P_{C} u_{\lambda}, u_{\lambda}-P_{C} u_{\lambda}\right)-\frac{1}{\lambda}\left\|u_{\lambda}-P_{C} u_{\lambda}\right\|^{2} \\
& \leq \frac{-1}{\lambda}\left\|u_{\lambda}-P_{C} u_{\lambda}\right\|^{2}
\end{aligned}
$$

\footnotetext{
${ }^{2} \mathrm{Si} C_{1}$ et $C_{2}$ sont des convexes fermés de $H$ on a : $P_{C_{1}} C_{2} \subset C_{2} \Longleftrightarrow P_{C_{2}} C_{1} \subset C_{1} \Longleftrightarrow$ $P_{C_{1}} C_{2}=P_{C_{2}} C_{1} \Longleftrightarrow P_{C_{1}} C_{2}=C_{1} \cap C_{2}$.
} 
on en déduit que $u_{\lambda} \in C$. On a alors

$$
\begin{aligned}
u_{\lambda}=(I+\lambda A)^{-1} u \Longleftrightarrow & \Phi\left(u_{\lambda}\right)+\frac{1}{\lambda}\left(u-u_{\lambda}, w-u_{\lambda}\right) \\
& \leq \Phi(w)+a\left(u_{\lambda}, w-u_{\lambda}\right) \quad \forall w \in D(\Phi) \\
\Longrightarrow & \left(\Phi+I_{C}\right)\left(u_{\lambda}\right)+\frac{1}{\lambda}\left(u-u_{\lambda}, w-u_{\lambda}\right) \\
& \leq\left(\Phi+I_{C}\right)(w)+a\left(u_{\lambda}, w-u_{\lambda}\right) \quad \forall w \in D(\Phi) \cap C \\
\Longleftrightarrow & u_{\lambda}=\left(I+\lambda A_{C}\right)^{-1} u
\end{aligned}
$$

où $A_{C}=A_{a, \Phi+I_{C}}$. On a donc, pour tout $u \in C$ et tout $\lambda>0,(I+$ $\lambda A)^{-1} u=\left(I+\lambda A_{C}\right)^{-1} u$, alors d'après la formule exponentielle $S(t) u=$ $\lim _{n \rightarrow \infty}\left(I+\frac{t}{n} A\right)^{-n} u$ pour tout $u \in H$ (cf. [2, Coroll. 4.4]) on en déduit (1.11).

3) $i v) \Rightarrow i$ ) est immédiat car $S_{C}(t)$ est à valeurs dans $\overline{D(\Phi) \cap C} \subset C$, et ii) $\Rightarrow$ iii) d'après (1.3).

On termine cette section par un théorème équivalent au théorème 1.1, d'un énoncé plus lourd mais qui nous sera très utile dans les sections suivantes.

Théorème 1.9. $\quad H, V, a, \Phi$ sont comme au début de la section, $(V, a, \Phi)$ vérifiant les hypothèses (1.1) à (1.6); $S(t)=S_{a, \Phi}(t), S_{\Gamma}(t)=S_{a, \Phi+I_{\Gamma}}(t)$ pour tout convexe fermé $\Gamma$ de $H$. Soient $C_{0}$ et $C$ deux convexes fermés de $H$ vérifiant,

$$
\text { pour tout } u \in C_{0}, \quad P_{C} u \in C_{0} .
$$

On suppose $C_{0}$ invariant par $S(t)$ c'est-à-dire,

$$
\text { pour tout } u \in C_{0} \quad \text { et tout } t \geq 0, \quad S(t) u \in C_{0} .
$$

Alors les assertions suivantes sont équivalentes,

i) Pour tout $u \in C \cap C_{0}$, et tout $t \geq 0, \quad S(t) u \in C$;

ii) Pour tout $u \in D(\Phi) \cap C_{0}, P_{C} u \in D(\Phi)$ et $\Phi\left(P_{C} u\right) \leq \Phi(u)+a\left(P_{C} u, u-\right.$ $\left.P_{C} u\right)$

iii) Pour tout $u \in D(\Phi) \cap C_{0}, \quad P_{C} u \in D(\Phi) \quad$ et $\Phi\left(P_{C} u\right) \leq \Phi(u)+a(u, u-$ $\left.P_{C} u\right)$;

iv) Pour tout $u \in C \cap C_{0}$ et tout $t \geq 0, \quad S(t) u=S_{C \cap C_{0}}(t) u$.

Preuve. Il est clair que le théorème 1.1 est un cas particulier du théorème 1.9 en prenant $C_{0}=H$. Le théorème 1.9 est une conséquence immédiate $\mathrm{du}$ théorème 1.1 en remarquant d'une part que (1.20) implique que i) est équivalent à, $S_{C_{0}}(t) u \in C \cap C_{0}$ pour tout $u \in C \cap C_{0}$, et tout $t \geq 0$, d'autre part (1.19) implique que,

$$
P_{C \cap C_{0}} u=P_{C} u \quad \text { pour } u \in C_{0} \text {. }
$$

\section{Application À la COMPARAison DE SEMi-Groupes}

Dans cette section $H=L^{2}(\Omega, \mathcal{B}, \mu ; \mathbb{R})$ muni du produit scalaire $(u, v)=$ $\int u v d \mu,(\Omega, \mathcal{B}, \mu)$ étant un espace mesuré. 
On se donne $V$ et $W$ des sous-espaces vectoriels de $H, a: V \times V \rightarrow \mathbb{R}, b$ : $W \times W \rightarrow \mathbb{R}, \Phi: V \rightarrow]-\infty,+\infty], \Psi: W \rightarrow]-\infty,+\infty]$ et l'on suppose que $(V, a, \Phi)$ et $(W, b, \Psi)$ vérifient les hypothèses (1.1) à (1.6). Les notations sont celles de I.

\section{1) Résultats.}

On a le critère de comparaison suivant:

Théorème 2.1. Les notations et hypothèses sont celles qui précèdent. On note $S(t)=S_{a, \Phi}(t)$ et $T(t)=S_{b, \Psi}(t)$. On se donne $\Gamma$ un convexe fermé de $H$ vérifiant

$$
\text { pour tout } v, w \in \Gamma, \quad v \wedge w \in \Gamma \text { et } v \vee w \in \Gamma \text {. }
$$

On suppose que $\Gamma$ est invariant par $S(t)$ et $T(t)$ c'est-à-dire

$$
\text { pour tout } u \in \Gamma \text { et tout } t \geq 0 \quad S(t) u \in \Gamma \text { et } T(t) u \in \Gamma \text {. }
$$

Les assertions suivantes sont équivalentes,

i) Pour tout $v, w$ dans $\Gamma$ tels que $v \leq w$ et pour tout $t \geq 0, S(t) v \leq T(t) w$;

ii) Pour tout $v \in D(\Phi) \cap \Gamma$ et $w \in D(\Psi) \cap \Gamma, v \wedge w \in D(\Phi), v \vee w \in D(\Psi)$ et

$$
\Phi(v \wedge w)+\Psi(v \vee w) \leq \Phi(v)+\Psi(w)+a\left(v,(v-w)^{+}\right)-b\left(w,(v-w)^{+}\right.
$$

En remplaçant $T(t)$ par $S(t)$, on a le corollaire immédiat suivant,

Corollaire 2.2. Soit $S(t)=S_{a, \Phi}(t)$ et soit $\Gamma$ un convexe fermé de $H$. On dit que $S(t)$ est croissant sur $\Gamma$ si pour tout $v, \hat{v} \in \Gamma$ tels que $v \leq \hat{v}$ et tout $t \geq 0, S(t) v \leq S(t) \hat{v}$.

On suppose que $\Gamma$ vérifie (2.1) et est invariant par $S(t)$. Alors, $S(t)$ est croissant sur $\Gamma$ si et seulement si, pour tout $v, \hat{v} \in D(\Phi) \cap \Gamma, v \wedge \hat{v}, v \vee \hat{v} \in$ $D(\Phi)$ et

$$
\Phi(v \wedge \hat{v})+\Phi(v \vee \hat{v}) \leq \Phi(v)+\Phi(\hat{v})+a\left(v,(v-\hat{v})^{+}\right)-a\left(\hat{v},(v-\hat{v})^{+}\right) .
$$

Remarque 2.3. Le théorème 2.1 nous permet de comparer $S(t)$ et $T(t)$ sur $\Gamma$ en remarquant que,

1) La propriété i) implique que pour tout $t \geq 0, S(t) \leq T(t)$ sur $\Gamma$ dans le sens $S(t) u \leq T(t) u$ pour tout $u$ dans $\Gamma$.

2) Si l'on suppose $S(t)$ ou $T(t)$ croissant sur $\Gamma$ alors la réciproque est vraie, plus précisément, $S(t) \leq T(t)$ sur $\Gamma$ pour tout $t \geq 0$ si et seulement si i) ou ii) du théorème 2.1 est vérifiée.

Corollaire 2.4. On suppose $\Phi=0$ et $\Psi=0$ et l'on note $S(t)=S_{a, 0}(t), T(t)$ $=S_{b, 0}(t)$. On suppose de plus que $S(t)$ est croissant sur $H$ ou $T(t)$ est croissant sur $H$. Alors les assertions suivantes sont équivalentes:

i) Pour tout $u \in H$ et tout $t \geq 0, S(t) u \leq T(t) u$;

ii) $V=W$, pour tout $v \in V$ on a $v^{+} \in V$ et

$$
b(v, w) \leq a(v, w) \quad \text { pour tout } \quad v \in V, w \in V \cap H^{+} .
$$


Preuve du corollaire 2.4. D'après la remarque 2.3. i) du corollaire 2.4. est équivalent à ii) du théorème 2.1 avec $\Gamma=H$, qui se traduit ici par: $V=W$, pour tout $v \in V$ on a $v^{+} \in V$ et

$$
b\left(\hat{v},(v-\hat{v})^{+}\right) \leq a\left(v,(v-\hat{v})^{+}\right) \quad \text { pour tout } \quad v, \hat{v} \in V
$$

Pour $v \in V$ et $w \in V \cap H^{+}$on prend $\hat{v}=v-t w$ avec $t>0$; en utilisant (1.2) et l'hémicontinuité (1.4), en faisant $t \rightarrow 0^{+}$on obtient que (2.6) implique (2.5). On suppose $(2.5)$; on prend $v, \hat{v} \in V$ et $w=(v-\hat{v})^{+}$on obtient d'après $(2.4)$,

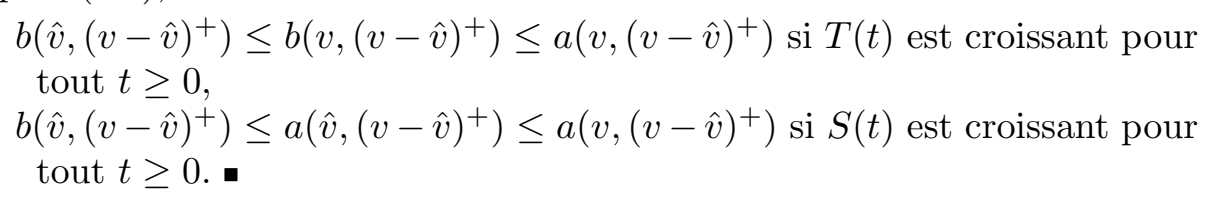

\section{2) Démonstrations.}

Pour démontrer le théorème 2.1 on se ramène à un résultat plus général qui sera en partie une conséquence immédiate du thèorème 1.9. et qui nous servira dans la section suivante sur la domination.

Dans la proposition suivante $H$ est un espace de Hilbert quelconque, $V, W$ des sous-espaces vectoriels de $H, a: V \times V \rightarrow \mathbb{R}, b: W \times W \rightarrow \mathbb{R}, \Phi:$ $V \rightarrow]-\infty,+\infty], \Psi: W \rightarrow]-\infty,+\infty]$; on suppose que $(V, a, \phi)$ et $(W, b, \psi)$ vérifient les hypothèses (1.1) à (1.6).

On se place dans l'espace de Hilbert $\mathcal{H}=H \times H$ muni du produit scalaire $((v, w),(\hat{v}, \hat{w}))_{\mathcal{H}}=(v, \hat{v})_{H}+(w, \hat{w})_{H}$. On note

$\mathcal{V}=V \times W$ muni de la norme $\|(v, w)\|_{\mathcal{V}}=\left(\|v\|_{V}^{2}+\|w\|_{W}^{2}\right)^{\frac{1}{2}}$,

$\mathcal{Q}: \mathcal{V} \rightarrow]-\infty,+\infty]$ définie par $\mathcal{Q}(v, w)=\Phi(v)+\Psi(w)$ avec $D(\mathcal{Q})=$ $D(\Phi) \times D(\Psi)$

$\mathcal{A}: \mathcal{V} \times \mathcal{V} \rightarrow \mathbb{R}$ définie par $\mathcal{A}((v, w),(\hat{v}, \hat{w}))=a(v, \hat{v})+b(w, \hat{w})$.

Il est clair que $(\mathcal{V}, \mathcal{A}, \mathcal{Q})$ vérifie les hypothèses $(1.1)$ à (1.6).

Si $\mathcal{S}(t)$ est le semi-groupe sur $\mathcal{H}$ associé à $(\mathcal{V}, \mathcal{A}, \mathcal{Q})$ défini par $(1.8)$ on a

$$
\mathcal{S}(t)(v, w)=\left(S_{a, \Phi}(t) v, S_{b, \Psi}(t) w\right) \quad \text { pour tout } \quad(v, w) \in \mathcal{H}
$$

Proposition 2.5. Les hypothèses et notations sont celles qui précèdent. On se donne $\mathcal{C}_{0}$ et $\mathcal{C}$ deux convexes fermés de $\mathcal{H}$ et deux applications $\alpha$ et $\beta$ de $\mathcal{C}_{0}$ dans $H$ vérifiant,

$$
\left\{\begin{array}{l}
\text { pour tout } t, s \in[0,1] \quad \text { avec } t+s \leq 1 \quad \text { et tout } \quad(v, w) \in \mathcal{C}_{0} \\
\left(v_{t}, w_{s}\right):=((1-t) v+t \alpha(v, w),(1-s) w+s \beta(v, w)) \in \mathcal{C}_{0} \quad \text { et } \\
\alpha\left(v_{t}, w_{s}\right)=v_{1-s}, \quad \beta\left(v_{t}, w_{s}\right)=w_{1-t} ; \\
\text { pour tout }(v, w) \in \mathcal{C}_{0}, P_{\mathcal{C}}(v, w)=\left(v_{\frac{1}{2}}, w_{\frac{1}{2}}\right) .
\end{array}\right.
$$

On suppose $\mathcal{C}_{0}$ invariant par $\mathcal{S}(t)$ c'est-à-dire pour tout $(v, w) \in \mathcal{C}_{0}$ et tout $t \geq 0 \mathcal{S}(t)(v, w) \in \mathcal{C}_{0}$. Alors, les propriétés suivantes sont équivalentes,

i) Pour tout $(v, w) \in \mathcal{C}_{0} \cap \mathcal{C}$ et tout $t \geq 0, \mathcal{S}(t)(v, w) \in \mathcal{C}$; 
ii) Pour tout $(v, w) \in D(\Phi) \times D(\Psi) \cap \mathcal{C}_{0}, \alpha(v, w) \in D(\Phi), \beta(v, w) \in D(\Psi)$ et

$$
\begin{aligned}
& \Phi(\alpha(v, w))+\Psi(\beta(v, w)) \\
& \quad \leq \Phi(v)+\Psi(w)+a(v, v-\alpha(v, w))+b(w, w-\beta(v, w)) .
\end{aligned}
$$

Preuve du théorème 2.1. On applique la proposition 2.5 avec $\mathcal{C}_{0}=\Gamma \times \Gamma, \mathcal{C}=$ $\{(v, w) \in \mathcal{H} ; v \leq w\}, \alpha(v, w)=v \wedge w, \beta(v, w)=v \vee w$ en remarquant que pour tout $(v, w) \in \mathcal{H}$,

$P_{\mathcal{C}}(v, w)=\left(\frac{1}{2} v+\frac{1}{2} v \wedge w, \frac{1}{2} w+\frac{1}{2} v \vee w\right), v-\alpha(v, w)=-(w-\beta(v, w))=(v-w)^{+}$.

Il reste à montrer (2.8): soient $v, w \in \Gamma, t \in[0,1]$; l'hypothèse $(2.1)$ et la convexité de $\Gamma$ impliquent que

$$
v_{t}=(1-t) v+t(v \wedge w) \in \Gamma \quad w_{t}=(1-t) w+t(v \vee w) \in \Gamma .
$$

Soient $t, s \in[0,1]$ avec $t+s \leq 1$,

$$
\begin{gathered}
v_{t}-w_{s}=v-w-(t+s)(v-w)^{+}=(1-(t+s))(v-w)^{+}-(v-w)^{-} \\
\Rightarrow\left(v_{t}-w_{s}\right)^{+}=(1-(t+s))(v-w)^{+}
\end{gathered}
$$

d'où

$$
\begin{aligned}
& \alpha\left(v_{t}, w_{s}\right)=v_{t} \wedge w_{s}=v_{t}-\left(v_{t}-w_{s}\right)^{+}=v-(1-s)(v-w)^{+}=v_{1-s} \\
& \beta\left(v_{t}, w_{s}\right)=v_{t} \vee w_{s}=w_{s}+\left(v_{t}-w_{s}\right)^{+}=w+(1-t)(v-w)^{+}=w_{1-t}
\end{aligned}
$$

Preuve de la proposition 2.5. On applique le théorème 1.9. avec $\mathcal{H}, \mathcal{V}$, $\mathcal{A}, \mathcal{Q}, \mathcal{C}_{0}, \mathcal{C}$

Alors i) de la proposition 2.5 est équivalente à

$$
\left\{\begin{array}{c}
\operatorname{pour} \operatorname{tout}(v, w) \in D(\Phi) \times D(\Psi) \cap \mathcal{C}_{0}, \alpha(v, w) \in V, \beta(v, w) \in W \\
\Phi\left(\frac{1}{2} v+\frac{1}{2} \alpha(v, w)\right)+\Psi\left(\frac{1}{2} w+\frac{1}{2} \beta(v, w)\right) \leq \Phi(v)+\Psi(w)+ \\
+a\left(v, \frac{v-\alpha(v, w)}{2}\right)+b\left(w, \frac{w-\beta(v, w)}{2}\right)
\end{array}\right.
$$

L'équivalence de (2.11) et (2.10) résulte du lemme suivant:

Lemme 2.6. $\quad H, V, W, a, b, \Phi, \Psi, \mathcal{C}_{0}$ sont comme dans la proposition 2.5 avec (2.8) vérifiée. Pour $t \in[0,1]$ on considère,

$$
\left\{\begin{aligned}
& \text { Pour tout }(v, w) \in D(\Phi) \times D(\Psi) \cap \mathcal{C}_{0}, \alpha(v, w) \in V, \beta(v, w) \in \text { Wet } \\
& \Phi\left(v_{t}\right)+\Psi\left(w_{t}\right) \leq \Phi(v)+\Psi(w) \\
&+t[a(v, v-\alpha(v, w))+b(w, w-\beta(v, w))] \\
& \text { avec } \quad v_{t}=(1-t) v+t \alpha(v, w), \quad w_{t}=(1-t) w+t \beta(v, w)
\end{aligned}\right.
$$

alors $\left(P_{1}\right),\left(P_{\frac{1}{2}}\right)$ et $\left(P_{t}\right)$ pour tout $t \in[0,1[$ sont équivalents.

Remarque 2.7. A part l'implication: $\left(P_{t}\right)$ pour tout $t \in\left[0,1\left[\Rightarrow\left(P_{1}\right)\right.\right.$, qui résulte de $\Phi$ et $\Psi$ s.c.i. ce lemme est un résultat de convexité dans lequel les propriétés topologiques des données n'interviennent pas ; on pourrait aussi remplacer $H \times H$ par $E \times F$ où $E$ et $F$ sont des espaces vectoriels. 
Preuve du lemme 2.6. On vient de remarquer que $\left(P_{t}\right)$ pour tout $t \in[0,1[\Rightarrow$ $\left(P_{1}\right) .\left(P_{1}\right) \Rightarrow\left(P_{t}\right)$ pour tout $t \in[0,1[$ résulte de la convexité de

$$
t \rightarrow \Phi\left(v_{t}\right)+\Psi\left(w_{t}\right)-t[a(v, v-\alpha(v, w))+b(w, w-\beta(v, w))] .
$$

Montrons que $\left(P_{\frac{1}{2}}\right) \Rightarrow\left(P_{t}\right)$ pour tout $t \in[0,1[$.

On suppose donc pour tout $(v, w) \in D(\Phi) \times D(\Psi) \cap C_{0}, \alpha(v, w) \in V, \beta(v, w) \in$ $W$ et $(2.11)$.

Fixons $(v, w) \in D(\Phi) \times D(\Psi) \cap \mathcal{C}_{0}$; on a donc $\alpha(v, w) \in V$ et $\beta(v, w) \in W$. Posons

$$
\varphi(t)=\Phi\left(v_{t}\right)+\Psi\left(w_{t}\right)-t[a(v, v-\alpha(v, w))+b(w, w-\beta(v, w))]
$$

$\varphi$ est convexe sur $[0,1]$.

On veut montrer que $\varphi(t) \leq \varphi(0) \quad \forall t \in[0,1[$. Soient $t, s \in[0,1]$ avec $t+s \leq 1$. On a d'après $(2.8)$,

$$
\left(v_{t}, w_{s}\right) \in \mathcal{C}_{0} \quad \text { et } \quad \alpha\left(v_{t}, w_{s}\right)=v_{1-s} \in V, \beta\left(v_{t}, w_{s}\right)=w_{1-t} \in W
$$

donc $\left(v_{t}, w_{s}\right)$ vérifie $(2.11)$ avec

$$
\begin{gathered}
\frac{1}{2} v_{t}+\frac{1}{2} \alpha\left(v_{t}, w_{s}\right)=v_{\frac{1+t-s}{2}}, \quad \frac{1}{2} w_{s}+\frac{1}{2} \beta\left(v_{t}, w_{s}\right)=w_{\frac{1+s-t}{2}}, \\
v_{t}-\alpha\left(v_{t}, w_{s}\right)=(1-t-s)(v-\alpha(v, w)), \quad w_{s}-\beta\left(v_{t}, w_{s}\right)=(1-t-s)(w-\beta(v, w)), \\
v-v_{t}=t(v-\alpha(v, w)) \Rightarrow a\left(v_{t}, v-\alpha(v, w)\right) \leq a(v, v-\alpha(v, w)), \\
w-w_{s}=s(w-\beta(v, w)) \Rightarrow b\left(w_{s}, w-\beta(v, w)\right) \leq b(w, w-\beta(v, w)),
\end{gathered}
$$

d'où

$$
\begin{aligned}
\Phi\left(v_{\frac{1+t-s}{2}}\right)+\Psi\left(w_{\frac{1+s-t}{2}}\right) & -\frac{1-t-s}{2}[a(v, v-\alpha(v, w))+b(w, w-\beta(v, w))] \\
& \leq \Phi\left(v_{t}\right)+\Psi\left(w_{s}\right) .
\end{aligned}
$$

En inversant le rôle de $t$ et $s$ et en additionnant on obtient

$$
\varphi\left(\frac{1+t-s}{2}\right)+\varphi\left(\frac{1+s-t}{2}\right) \leq \varphi(t)+\varphi(s) .
$$

On en conclut que $\varphi(t) \leq \varphi(0)$ par application du lemme suivant:

Lemme 2.8. Soit $\varphi:[0,1[\rightarrow]-\infty,+\infty]$ convexe, alors

$$
\begin{aligned}
& \varphi\left(\frac{1+t-s}{2}\right)+\varphi\left(\frac{1+s-t}{2}\right) \leq \varphi(t)+\varphi(s) \forall t, s \in[0,1[\text { avec } t+s \leq 1 \\
& \Rightarrow \varphi(t) \leq \varphi(0) \quad \text { pour tout } t \in[0,1[.
\end{aligned}
$$

Preuve du lemme 2.8. Le lemme étant trivial si $\varphi(0)=+\infty$ on suppose $\varphi(0)<\infty$.

1) Faisant $s=t=0$ dans $(2.12)$ on a $\varphi\left(\frac{1}{2}\right) \leq \varphi(0)$, et par convexité $\varphi(t) \leq \varphi(0)$ pour tout $t \in\left[0, \frac{1}{2}\right]$ 
2) $s=0$ dans (2.12) implique

$\varphi\left(\frac{1+t}{2}\right)+\varphi\left(\frac{1-t}{2}\right) \leq \varphi(t)+\varphi(0) \quad \forall t \in[0,1]$ avec $\varphi\left(\frac{1-t}{2}\right)<\infty$ car $\frac{1-t}{2} \leq \frac{1}{2}$. On en déduit que si $\left(t_{n}\right)$ est définie par $t_{0} \in\left[0, \frac{1}{2}\right], t_{n}=$ $\frac{1+t_{n-1}}{2}$ alors $\varphi\left(t_{n}\right)<\infty$ pour tout $n$; comme $t_{n} \rightarrow 1$ on en conclut que $\varphi(t)<\infty$ pour tout $t \in[0,1[$.

3) Après changement de variables (2.12) devient

$$
\begin{aligned}
\varphi\left(\frac{1}{2}+h\right)+\varphi\left(\frac{1}{2}-h\right) & \leq \varphi(k+h)+\varphi(k-h) \\
& \text { pour tout } h, k \text { tels que } 0 \leq h \leq k \leq \frac{1}{2}, k+h<1 .
\end{aligned}
$$

Pour $h$ fixé dans $\left[0, \frac{1}{2}\left[, \alpha_{h}: k \in\left[h, \frac{1}{2}[\rightarrow \varphi(k+h)+\varphi(k-h) \in \mathbb{R}\right.\right.\right.$ est convexe. D'après $(2.13)$ on a $\alpha_{h}\left(\frac{1}{2}\right) \leq \alpha_{h}(k) \forall k \in\left[h, \frac{1}{2}[\right.$, par convexité on en déduit que $\alpha_{h}$ est décroissante sur $\left[h, \frac{1}{2}\left[\right.\right.$ d'où $\varphi_{d}^{\prime}(k+h)+\varphi_{d}^{\prime}(k-h) \leq 0$ pour tout $k \in\left[h, \frac{1}{2}\left[\right.\right.$ et $h \in\left[0, \frac{1}{2}[\right.$.

Pour $k$ fixé dans $\left[0, \frac{1}{2}[\right.$ on en déduit que $h \in[0, k] \rightarrow \varphi(k+h)-\varphi(k-h)$ est décroissante, en particulier $\varphi(2 k) \leq \varphi(0)$.

3) Comparaison sur $H^{+}=L^{2}\left(\Omega, \mathcal{B}, \mu ; \mathbb{R}^{+}\right)$.

On termine cette section en montrant comment le théorème 2.1 étend un résultat de E. M. Ouhabaz sur la "domination positive" ou "comparaison sur les positifs" ([8]). On rappelle (cf. Exemple 1.3) que $S(t)$ est positif si pour tout $u \in H^{+}$et tout $t \geq 0, S(t) u \in H^{+} . V$ est un idéal positif de $W$ si

1) $V \subset W$

2) Pour tout $v \in V \cap H^{+}, w \in W \cap H^{+}$tels que $w \leq v, w \in V$.

Corollaire 2.9. On suppose $\Phi=0$ et $\Psi=0$ et l'on pose $S(t)=S_{a, 0}(t), T(t)$ $=S_{b, 0}(t)$. On suppose de plus que $S(t)$ et $T(t)$ sont positifs et $T(t)$ est croissant sur $H^{+}$. Alors les assertions suivantes sont équivalentes:

i) Pour tout $u \in H^{+}$et tout $t \geq 0, S(t) u \leq T(t) u$;

ii) $V$ est un idéal positif de $W$ et

$$
b(v, \hat{v}) \leq a(v, \hat{v}) \quad \text { pour tout } v, \hat{v} \in V \cap H^{+} ;
$$

iii) $V$ est un idéal positif de $W$ et

(2.15) $b(v, w) \leq a(v, w)$ pour tout $v \in V \cap H^{+}, w \in W \cap H^{+}$avec $w \leq v$. 
Preuve. Compte tenu de la croissance de $T(t)$ sur $H^{+}$, i) du corollaire (2.9) est équivalente à ii) du théorème 2.1 avec $\Gamma=H^{+}$, qui se traduit par $V$ est un idéal positif de $W$ et

$$
b\left(w,(v-w)^{+}\right) \leq a\left(v,(v-w)^{+}\right) \text {pour } v \in V \cap H^{+} \text {et } w \in W \cap H^{+} .
$$

On suppose que $V$ est un idéal positif de $W$. Soient $v, \hat{v} \in V \cap H^{+}$, pour $t>0, v_{t}=v+t \hat{v} \in V \cap H^{+}$. Si (2.16) est vérifiée alors $b(v, \hat{v}) \leq a(v+t \hat{v}, \hat{v})$; on fait $t \rightarrow 0^{+}$en utilisant l'hémicontinuité (1.4) on obtient (2.14). Soient $v \in V \cap H^{+}, w \in W \cap H^{+},(v-w)^{+} \in W \cap H^{+}$et $(v-w)^{+} \leq v$, donc si (2.15) est vérifiée alors on a

$$
b\left(v,(v-w)^{+}\right) \leq a\left(v,(v-w)^{+}\right) .
$$

Maintenant $T(t)$ étant croissant sur $H^{+}$, toujours d'après le théorème 2.1 avec $\Gamma=H^{+}, S(t)=T(t)$, on a pour tout $v, w \in W \cap H^{+}$

$$
b\left(w,(v-w)^{+}\right) \leq b\left(v,(v-w)^{+}\right) .
$$

(2.17) et (2.18) impliquent (2.16).

\section{Applichtion À la domination De SEMI-Groupes}

Soit $H=L^{2}(\Omega, \mathcal{B}, \mu ; \mathbb{K})$ où $(\Omega, \mathcal{B}, \mu)$ est un espace mesuré $\sigma$-fini, $\mathbb{K}=\mathbb{R}$ ou $\mathbb{C} ; \mathbb{H}$ est muni du produit scalaire réel $(u, v)_{H}=\operatorname{Re} \int u \bar{v} d \mu$.

On notera $H_{\mathbb{R}}=L^{2}(\Omega, \mathcal{B}, \mu ; \mathbb{R})$ et $H_{\mathbb{R}}^{+}$le cône convexe fermé de $H$ formé des éléments positifs de $H_{\mathbb{R}}$.

Pour $u \in H$, sign $u$ est défini comme dans l'exemple 1.5.

On se donne $V$ et $W$ des sous-espaces vectoriels de $H, a: V \times V \rightarrow \mathbb{R}, b$ : $W \times W \rightarrow \mathbb{R}, \Phi: V \rightarrow]-\infty, \infty], \Psi: W \rightarrow]-\infty, \infty]$ et l'on suppose que $(V, a, \Phi)$ et $(W, b, \Psi)$ vérifient les hypothèses (1.1) à (1.6).

On note $S(t)=S_{a, \Phi}(t)$ et $T(t)=S_{b, \Psi}(t)$ les semi-groupes sur $H$ associés à $(V, a, \Phi)$ et $(W, b, \Psi)$ respectivement, définis par (1.8). On s'intéresse à la domination de $S(t)$ par $T(t)$ avec

Définitions 3.1 On dit que $T(t)$ est positif si pour tout $u \in H_{\mathbb{R}}^{+}$et tout $t \geq 0, T(t) u \in H_{\mathbb{R}}^{+}$. On dit que $S(t)$ est dominé par $T(t)$ si $T(t)$ est positif et

$$
|S(t) u| \leq T(t)|u| \text { pour tout } u \in H \text { et tout } t \geq 0 .
$$

Remarque 3.2. Pour $u \in H \quad P_{H_{\mathbb{R}}^{+}} u=(R e u)^{+}$, donc d'après le théorème 1.1 si $T(t)$ est positif, pour tout $w \in D(\Psi),(\operatorname{Re} w)^{+} \in D(\Psi)$.

Théorème 3.3. Soient $S(t)=S_{a, \Phi}(t), T(t)=S_{b, \Psi}(t)$. On suppose $T(t)$ positif et croissant sur $H_{\mathbb{R}}^{+}$, alors les assertions suivantes sont équivalentes:

i) $S(t)$ est dominé par $T(t)$;

ii) Pour tout $v \in D(\Phi)$ et $w \in D(\Psi) \cap H_{\mathbb{R}}^{+},(|v| \wedge w)$ sign $v \in D(\Phi),|v| \vee w \in$ $D(\Psi)$ et

$$
\begin{aligned}
\Phi((|v| \wedge w) \operatorname{sign} v)+ & \Psi(|v| \vee w) \leq \Phi(v)+\Psi(w) \\
& +a\left(v,(|v|-w)^{+} \operatorname{sign} v\right)-b\left(w,(|v|-w)^{+}\right) .
\end{aligned}
$$


Comme pour la comparaison on se ramène à un résultat plus général de domination en faisant les remarques suivantes qui justifieront l'hypothèse de croissance faite sur $T(t)$.

\section{Remarque 3.4.}

1) Si pour tout $(v, w) \in H \times H_{\mathbb{R}}^{+}$tels que $|v| \leq w$ et tout $t \geq 0,|S(t) v| \leq$ $T(t) w$ alors $S(t)$ est dominé par $T(t)$;

2) Si $T(t)$ est croissant sur $H_{\mathbb{R}}^{+}$la réciproque est vraie.

Donc il suffit de montrer la

Proposition 3.5. $S(t)=S_{a, \Phi}(t), T(t)=S_{b, \Psi}(t)$. On suppose $T(t)$ positif alors les assertions suivantes sont équivalentes:

i) Pour tout $(v, w) \in H \times H_{\mathbb{R}}^{+}$tels que $|v| \leq w$ et tout $t \geq 0,|S(t) v| \leq$ $T(t) w$;

ii) Pour tout $v \in D(\Phi)$ et $w \in D(\Psi) \cap H_{\mathbb{R}}^{+},(|v| \wedge w)$ sign $v \in D(\Phi),|v| \vee w \in$ $D(\Psi)$ et $\Phi((|v| \wedge w) \operatorname{sign} v)+\Psi(|v| \vee w) \leq \Phi(v)+\Psi(w)+a\left(v,(|v|-w)^{+}\right.$ sign $v)-b\left(w,(|v|-w)^{+}\right)$.

Preuve. On applique la proposition 2.5 avec $\mathcal{C}_{0}=H \times H_{\mathbb{R}}^{+}, \mathcal{C}=\{(v, w) \in$ $\left.H \times H_{\mathbb{R}}^{+} ;|v| \leq w\right\}, \alpha(v, w)=(|v| \wedge w) \operatorname{sign} v, \beta(v, w)=|v| \vee w$ en remarquant que pour $(v, w) \in H \times H_{\mathbb{R}}^{+}$

$$
P_{\mathcal{C}}(v, w)=\left(\frac{1}{2} v+\frac{1}{2}(|v| \wedge w) \operatorname{sign} v, \frac{1}{2} w+\frac{1}{2}(|v| \vee w)\right) .
$$

Il reste à montrer $(2.8)$. Soient $(v, w) \in H \times H_{\mathbb{R}}^{+}, t \in[0,1]$; il est clair que,

$$
v_{t}=(1-t) v+t(|v| \wedge w) \operatorname{sign} v \in H, \quad w_{t}=(1-t) w+t(|v| \vee w) \in H_{\mathbb{R}}^{+}
$$

ensuite si $t, s \in[0,1]$ avec $t+s \leq 1$,

$\left|v_{t}\right|-w_{s}=|v|-w-(t+s)(|v|-w)^{+}=(1-(t+s))(|v|-w)^{+}-(|v|-w)^{-}$ ce qui implique

$$
\left(\left|v_{t}\right|-w_{s}\right)^{+}=(1-(t+s))(|v|-w)^{+} .
$$

Alors un calcul simple montre que $\alpha\left(v_{t}, w_{s}\right)=v_{1-s}$ et $\beta\left(v_{t}, w_{s}\right)=w_{1-t}$.

Montrons comment le théorème 3.3 étend le résultat de E. M. Ouhabaz sur la domination ([8]).

On dit de $V$ est un idéal de $W$ si

1) pour tout $v \in V,|v| \in W$;

2) pour tout $v \in V$ et $w \in W \cap H_{\mathbb{R}}^{+}$tels que $w \leq|v|$, wsign $v \in V$.

Corollaire 3.6. On suppose $\Phi=0$ et $\Psi=0$ et l'on pose $S(t)=S_{a, 0}(t)$, $T(t)=S_{b, 0}(t)$. On suppose $T(t)$ positif, réel et croissant sur $H_{\mathbb{R}}^{+}{ }^{3}$. Alors les assertions suivantes sont équivalentes:

i) $S(t)$ est dominé par $T(t)$;

\footnotetext{
${ }^{3} T(t)$ est réel si pour tout $w \in H_{\mathbb{R}}$ et tout $t \geq 0, T(t) w \in H_{\mathbb{R}} T(t)$ est croissant sur $H_{\mathbb{R}}^{+}$ si pour tout $w, \hat{w} \in H_{\mathbb{R}}^{+}$tels que $w \leq \hat{w}$ et pour tout $t \geq 0, T(t) w \leq T(t) \hat{w}$.

Si $T(t)$ est linéaire $T(t)$ positif implique $T(t)$ réel et $T(t)$ croissant.
} 
ii) $V$ est un idéal de $W$ et

(3.1) $b(|v|, w) \leq a(v$, wsign $v)$ pour tout $v \in V, w \in W \cap H_{\mathbb{R}}^{+}$avec $w \leq|v|$.

Preuve. Tout d'abord il est facile de vérifier que

$(|v| \wedge w) \operatorname{sign} v \in V$ et $|v| \vee w \in W \quad$ pour tout $v \in V$ et $w \in W \cap H_{\mathbb{R}}^{+}$

est équivalent à $V$ est un idéal de $W$. Donc d'après le théorème $(3.3), S(t)$ est dominé par $T(t)$ si et seulement si $V$ est un idéal de $W$ et

$$
\begin{aligned}
b\left(w,(|v|-w)^{+}\right) \leq & a\left(v,(|v|-w)^{+} \operatorname{sign} v\right) \\
& \text { pour tout } v \in V \text { et } w \in W \cap H_{\mathbb{R}}^{+} .
\end{aligned}
$$

Supposons que $V$ soit un idéal de $W$. Soit $v \in V$ et $w \in W \cap H_{\mathbb{R}}^{+}$avec $w \leq|v|$ alors pour tout $t \in] 0,1],|v|-t w \in W \cap H_{\mathbb{R}}^{+}$. Si (3.2) est vérifiée alors on a

$$
b(|v|-t w, w) \leq a(v, w \operatorname{signv}) .
$$

On fait $t \rightarrow 0^{+}$en utilisant l'hémicontinuité (1.4), on obtient (3.1).

Soient $v \in V$ et $w \in W \cap H_{\mathbb{R}}^{+}$alors $(|v|-w)^{+} \in W \cap H_{\mathbb{R}}^{+}$et $(|v|-w)^{+} \leq|v|$. Donc si (3.1) est vérifiée, on obtient

$$
b\left(|v|,(|v|-w)^{+}\right) \leq a\left(v,(|v|-w)^{+} \operatorname{sign} v\right) .
$$

$T(t)$ étant réel et positif on peut utiliser la proposition 2.5. avec $\Gamma=H_{\mathbb{R}}^{+}$ pour affirmer que $T(t)$ est croissant sur $H_{\mathbb{R}}$ si et seulement si pour tout $w, \hat{w} \in W \cap H_{\mathbb{R}}^{+}, w \wedge \hat{w}, w \vee \hat{w} \in W \cap H_{\mathbb{R}}^{+}$et

$$
b\left(w,(\hat{w}-w)^{+}\right) \leq b\left(\hat{w},(\hat{w}-w)^{+}\right) .
$$

(3.2) se déduit de (3.3) et (3.4).

\section{EXEMPLE 1}

Soient $H=L^{2}(] 0,1[)$ et $V=W^{1, p}(] 0,1[)$ avec $1<p<\infty$. On se donne, - $\left.\left(a_{0}, a_{1}\right):\right] 0,1\left[\times \mathbb{R}^{2} \longrightarrow \mathbb{R}^{2}\right.$ une application des variables $(x, k, \xi)$ que l'on suppose de Carathéodory et monotone en $(k, \xi)$ c'est-à- dire vérifiant,

$$
\begin{array}{r}
\left(a_{0}(\cdot, k, \xi), a_{1}(\cdot, k, \xi)\right) \text { est mesurable pour tout }(k, \xi) \in \mathbb{R}^{2} \\
\left.\left(a_{0}(x, \cdot, \cdot), a_{1}(x, \cdot, \cdot)\right) \text { est continue sur } \mathbb{R}^{2} \text { p.p. } x \in\right] 0,1[, \\
a_{0}(x, k, \xi)(k-\hat{k})+a_{1}(x, k, \xi)(\xi-\hat{\xi}) \\
\geq a_{0}(x, \hat{k}, \hat{\xi})(k-\hat{k})+a_{1}(x, \hat{k}, \hat{\xi})(\xi-\hat{\xi}) \\
\text { p.p. } x \in] 0,1\left[, \operatorname{pour} \text { tout }(k, \xi),(\hat{k}, \hat{\xi}) \in \mathbb{R}^{2}\right.
\end{array}
$$

on suppose de plus

$$
\begin{aligned}
\left|a_{0}(x, k, \xi)\right|+\left|a_{1}(x, k, \xi)\right|^{p^{\prime}} \leq & c_{0}(k)\left(c_{1}(x)+|\xi|^{p}\right) \\
& \text { p.p. } x \in] 0,1\left[, \text { pour tout }(k, \xi), \in \mathbb{R}^{2}\right.
\end{aligned}
$$

où $c_{0} \in \mathcal{C}(\mathbb{R}), c_{1} \in L^{1}(] 0,1[)$ et $p^{\prime}$ est le conjugué de $p$.

- $\left.\left.\varphi: \mathbb{R}^{2} \longrightarrow\right]-\infty, \infty\right]$ une application convexe, s.c.i de domaine $D(\varphi) \neq \emptyset$. 
On définit alors, $a: V \times V \longrightarrow \mathbb{R}$ et $\Phi: V \longrightarrow]-\infty, \infty]$ par $(4.4) a(u, v)=\int_{0}^{1}\left(a_{0}\left(x, u(x), u^{\prime}(x)\right) v(x)+a_{1}\left(x, u(x), u^{\prime}(x)\right) v^{\prime}(x)\right) d x$, (4.5) $\Phi(u)=\varphi(u(0), u(1))$.

Il est clair alors que $(V, a, \Phi)$ vérifie les hypothèses (1.1) à (1.5). Remarquons que $a$ est T-monotone c'est-à-dire

$$
a\left(u,(u-\hat{u})^{+}\right) \geq a\left(\hat{u},(u-\hat{u})^{+}\right) \quad \text { pour tout } u, \hat{u} \in V .
$$

En effet d'après (4.2) on a

$$
\begin{aligned}
a\left(u,(u-\hat{u})^{+}\right)-a\left(\hat{u},(u-\hat{u})^{+}\right)= & \int_{u>\hat{u}}\left[\left(a_{0}\left(\cdot, u, u^{\prime}\right)-a_{0}\left(\cdot, \hat{u}, \hat{u}^{\prime}\right)\right)(u-\hat{u})\right. \\
& \left.+\left(a_{1}\left(\cdot, u, u^{\prime}\right)-a_{1}\left(\cdot, \hat{u}, \hat{u}^{\prime}\right)\right)\left(u^{\prime}-\hat{u}^{\prime}\right)\right] \geq 0 .
\end{aligned}
$$

On suppose de plus que $(a, \Phi)$ vérifie l'hypothèse de coercivité (1.6). On note $S(t)=S_{a, \Phi}(t)$ le semi-groupe associé à $(V, a, \Phi)$ définie par (1.8). On a alors la

Proposition 4.1. $S(t)$ est croissant sur $H$ si et seulement si ou bien il existe $\left(e_{0}, e_{1}\right) \in\left[0, \infty\left[{ }^{2},\left(u_{0}, u_{1}\right) \in \mathbb{R}^{2}\right.\right.$ tels que

$$
D(\varphi) \subset\left\{\left(u_{0}, u_{1}\right)+t\left(e_{0}, e_{1}\right), t \in \mathbb{R}\right\}
$$

ou bien $\operatorname{int} D(\varphi) \neq \emptyset, \frac{\partial^{2} \varphi}{\partial x_{0} \partial x_{1}} \leq 0$ dans $\mathcal{D}^{\prime}(\operatorname{int} D(\varphi))$, et pour tout $\left(x_{0}, x_{1}\right)$, $\left(y_{0}, y_{1}\right) \in D(\varphi)$ tels que $\left(x_{0}-y_{0}\right)\left(x_{1}-y_{1}\right)<0,\left(x_{0}, y_{1}\right),\left(y_{0}, x_{1}\right) \in D(\varphi)$.

Preuve. D'après le corollaire 2.2, $S(t)$ est croissant si et seulement si pour tout $\left(x_{0}, x_{1}\right) \in \mathbb{R}^{2},\left(y_{0}, y_{1}\right) \in \mathbb{R}^{2}, v, w \in V$ avec $(v(0), v(1))=\left(y_{0}, y_{1}\right)$, $(w(0), w(1))=\left(x_{0}, x_{1}\right)$ on a

$$
\begin{aligned}
& \varphi\left(x_{0} \wedge y_{0}, x_{1} \wedge y_{1}\right)+\varphi\left(x_{0} \vee y_{0}, x_{1} \vee y_{1}\right) \\
& \leq \varphi\left(x_{0}, x_{1}\right)+\varphi\left(y_{0}, y_{1}\right)+a\left(v,(v-w)^{+}\right)-a\left(w,(v-w)^{+}\right)
\end{aligned}
$$

or d'après $(4.6)$ si $\left(x_{0}, x_{1}\right)$ et $\left(y_{0}, y_{1}\right)$ sont comparables (4.7) est vérifiée.

D'autre part si $\operatorname{int} D(\varphi)$ est vide et si $S(t)$ est croissant alors, d'après (4.7), tous les éléments de $D(\varphi)$ sont comparables et donc

$$
D(\varphi) \subset\left\{\left(u_{0}, u_{1}\right)+t\left(e_{0}, e_{1}\right) ; t \in \mathbb{R}\right\} \quad \text { avec }\left(e_{0}, e_{1}\right) \in\left[0, \infty\left[^{2} .\right.\right.
$$

On suppose désormais $U:=\operatorname{int} D(\varphi) \neq \emptyset$. D'après les remarques précédentes on a, $S(t)$ est croissant si et seulement si pour tout $\left(x_{0}, x_{1}\right),\left(y_{0}, y_{1}\right) \in \mathbb{R}^{2}$ avec $\left(x_{0}-y_{0}\right)\left(x_{1}-y_{1}\right)<0$, tout $v, w \in V$ avec $(v(0), v(1))=\left(y_{0}, y_{1}\right),(w(0), w(1))$ $=\left(x_{0}, x_{1}\right)$,

$$
\begin{aligned}
\varphi\left(x_{0}, y_{1}\right)+\varphi\left(y_{0}, x_{1}\right) \leq & \varphi\left(x_{0}, x_{1}\right)+\varphi\left(y_{0}, y_{1}\right) \\
& +a\left(v,(v-w)^{+}\right)-a\left(w,(v-w)^{+}\right) .
\end{aligned}
$$

Supposons (4.8). Soient $(h, k) \in] 0, \infty\left[{ }^{2}\right.$ et $\zeta \in \mathcal{D}\left(\mathbb{R}^{2}\right)$ avec $\zeta \geq 0$ et $\operatorname{supp} \zeta \subset$ $U$. On utilise $(4.8)$ avec $\left(x_{0}, x_{1}\right) \in \mathbb{R}^{2},\left(y_{0}, y_{1}\right)=\left(x_{0}-t h, x_{1}+t k\right) t>0$,

$$
w(x)=x_{0}(1-x)+x_{1} x \text { pour } x \in[0,1]
$$


on obtient,

$$
v(x)= \begin{cases}\left(x_{0}-t h\right)(1-x)+\left(x_{1}+t h\right) x & \text { si } 0 \leq x \leq \frac{1}{2}, \\ \left(x_{0}-t k\right)(1-x)+\left(x_{1}+t k\right) x & \text { si } \frac{1}{2} \leq x \leq 1\end{cases}
$$

$$
\begin{array}{r}
\varphi\left(x_{0}, x_{1}+t k\right)+\varphi\left(x_{0}-t h, x_{1}\right) \leq \varphi\left(x_{0}, x_{1}\right)+\varphi\left(x_{0}-t h, x_{1}+t k\right) \\
+2 t k\left(\gamma\left(x_{0}-t k, x_{1}+t k\right)-\gamma\left(x_{0}, x_{1}\right)\right)
\end{array}
$$

avec pour $\left(z_{0}, z_{1}\right) \in \mathbb{R}^{2}$

$$
\begin{aligned}
\gamma\left(z_{0}, z_{1}\right)=\int_{\frac{1}{2}}^{1}[ & a_{0}\left(x, z_{0}(1-x)+z_{1} x, z_{1}-z_{0}\right)\left(x-\frac{1}{2}\right) \\
& \left.+a_{1}\left(x, z_{0}(1-x)+z_{1} x, z_{1}-z_{0}\right)\right] d x
\end{aligned}
$$

$\gamma$ est continue sur $\mathbb{R}^{2}$ et $\varphi$ est continue sur $U$, de plus si $\left(x_{0}, x_{1}\right) \in \operatorname{supp} \zeta$ et si

$$
0<t<\frac{\operatorname{dist}(\operatorname{supp} \zeta, \partial U)}{2\|(h, k)\|}
$$

alors $\left(x_{0}-t h, x_{1}+t k\right) \in U$, donc en multipliant (4.9) par $\zeta\left(x_{0}, x_{1}\right)$ et en intégrant en $\left(x_{0}, x_{1}\right)$ sur $\mathbb{R}^{2}$ on obtient après des changements de variables convenables,

$$
\begin{aligned}
\int_{\Omega} \varphi\left(x_{0}, x_{1}\right)[\zeta & \left(x_{0}, x_{1}-t k\right)+\zeta\left(x_{0}+t h, x_{1}\right) \\
& \left.-\zeta\left(x_{0}, x_{1}\right)-\zeta\left(x_{0}+t h, x_{1}-t k\right)\right] d x_{0} d x_{1} \\
\leq & 2 t k \int_{\mathbb{R}^{2}} \gamma\left(x_{0}, x_{1}\right)\left[\zeta\left(x_{0}+t k, x_{1}-t k\right)-\zeta\left(x_{0}, x_{1}\right)\right] d x_{0} d x_{1}
\end{aligned}
$$

on divise par $t^{2}$ et l'on fait $t \rightarrow 0^{+}$, il vient que,

$$
\begin{aligned}
\int_{\Omega} \varphi\left(x_{0}, x_{1}\right) & \frac{\partial^{2} \zeta}{\partial x_{0} \partial x_{1}}\left(x_{0}, x_{1}\right) d x_{0} d x_{1} \\
& \leq \frac{2 k}{h} \int_{\mathbb{R}^{2}} \gamma\left(x_{0}, x_{1}\right)\left(\frac{\partial \zeta}{\partial x_{0}}\left(x_{0}, x_{1}\right)-\frac{\partial \zeta}{\partial x_{1}}\left(x_{0}, x_{1}\right)\right) d x_{0} d x_{1}
\end{aligned}
$$

en faisant $k \rightarrow 0$ on obtient que $\frac{\partial^{2} \varphi}{\partial x_{0} \partial x_{1}} \leq 0$ dans $\mathcal{D}^{\prime}(U)$.

Pour la réciproque il suffit, d'après (4.6), d'appliquer le lemme suivant,

Lemme 4.2. Soit $\left.\left.\varphi: \mathbb{R}^{2} \longrightarrow\right]-\infty, \infty\right]$ convexe s.c.i telle que $U=\operatorname{int} D(\varphi)$ $\neq \emptyset$; alors les propriétés suivantes sont équivalentes:

i) pour tout $\left(x_{0}, x_{1}\right),\left(y_{0}, y_{1}\right) \in \mathbb{R}^{2}$ avec $\left(x_{0}-y_{0}\right)\left(x_{1}-y_{1}\right)<0$

$$
\varphi\left(x_{0}, y_{1}\right)+\varphi\left(y_{0}, x_{1}\right) \leq \varphi\left(x_{0}, x_{1}\right)+\varphi\left(y_{0}, y_{1}\right)
$$

ii) $\frac{\partial^{2} \varphi}{\partial x_{0} \partial x_{1}} \leq 0$ dans $\mathcal{D}^{\prime}(U)$ et

$$
\begin{array}{r}
\text { pour tout }\left(x_{0}, x_{1}\right),\left(y_{0}, y_{1}\right) \in D(\varphi) \text { avec }\left(x_{0}-y_{0}\right)\left(x_{1}-y_{1}\right)<0, \\
\left(x_{0}, y_{1}\right),\left(y_{0}, y_{1}\right) \in D(\varphi) .
\end{array}
$$


Preuve: $i) \Rightarrow i i$ ) vient d'etre démontré en prenant $a_{0}=0$ et $a_{1}=0$. Pour montrer $i i) \Rightarrow i$ ) on se ramène par des techniques de régularisation habituelles à $\varphi$ de classe $\mathcal{C}^{1} \operatorname{sur} U$ d'où l'on déduit (4.10) pour $\left(x_{0}, x_{1}\right),\left(y_{0}, y_{1}\right)$ $\in U$ avec $\left(x_{0}-y_{0}\right)\left(x_{1}-y_{1}\right)<0$.

Maintenant si $\left(x_{0}, x_{1}\right),\left(y_{0}, y_{1}\right) \in D(\varphi)$ alors par convexité de $D(\varphi)$ et $(4.11)$ on a pour $t \in] 0,1[$

$$
\left(t\left(y_{0}-x_{0}\right)+x_{0}, t\left(y_{1}-x_{1}\right)+x_{1}\right),\left(t\left(x_{0}-y_{0}\right)+y_{0}, t\left(x_{1}-y_{1}\right)+y_{1}\right) \in U
$$

et donc

$$
\begin{aligned}
& \varphi\left(t\left(y_{0}-x_{0}\right)+x_{0}, t\left(x_{1}-y_{1}\right)+y_{1}\right)+\varphi\left(t\left(x_{0}-y_{0}\right)+y_{0}, t\left(y_{1}-x_{1}\right)+x_{1}\right) \\
& \leq \varphi\left(t\left(y_{0}-x_{0}\right)+x_{0}, t\left(y_{1}-x_{1}\right)+x_{1}\right)+\varphi\left(t\left(x_{0}-y_{0}\right)+y_{0}, t\left(x_{1}-y_{1}\right)+y_{1}\right)
\end{aligned}
$$

comme ces fonctions de $t$ sont convexes s.c.i et finies sur $[0,1]$, elles sont continues sur $[0,1]$, donc lorsque $t \rightarrow 0^{+}$on obtient (4.10).

\section{EXEMPLE 2}

Soient $H=L^{2}(\Omega)$ où $\Omega$ est un ouvert borné de $\mathbb{R}^{N}$ à frontière lipschitzienne, $V=W^{1, p}(\Omega) \cap L^{2}(\Omega)$ avec $1<p<\infty$, muni de la norme $\|u\|_{V}=\|u\|_{L^{2}}+\|D u\|_{L^{p}}$ où $D u$ est le gradient de $u$ (cette norme est équivalente à celle de $W^{1, p}(\Omega)$ pour $p \geq 2$ ).

On se donne,

- $\mu$ une mesure de Radon positive sur $\bar{\Omega}$, d'énergie finie relativement à $V$ (c'est-à-dire, il existe $C$ tel que $\int_{\bar{\Omega}}|u| d \mu \leq C\|u\|_{V}$ pour tout $u \in$ $W^{1, p}(\Omega) \cap \mathcal{C}(\bar{\Omega})$ ce qui est équivalent à $\mu$ ne charge pas les ensembles de $V$-capacité nulle (cf. [1]).

- $\varphi: \bar{\Omega} \times \mathbb{R} \longrightarrow[0, \infty]$ vérifiant,

(5.1) $\varphi(\cdot, h)$ est $\mu$-mesurable pour tout $h \in \mathbb{R}$

(5.2) $\varphi(x, \cdot)$ est convexe s.c.i pour $\mu$ p.p. $x \in \bar{\Omega}$

(5.3) $\varphi(\cdot, 0)=0$

on définit alors pour tout $u \in V$

$$
\Phi(u)=\int_{\bar{\Omega}} \varphi(x, \tilde{u}(x)) d \mu(x)
$$

où $\tilde{u}$ est un représentant quasi-continu de $u$ (défini quasi partout donc $\mu$ p.p.). $\Phi$ est alors convexe s.c.i de $V$ dans $[0, \infty]$ avec $\Phi(0)=0(\operatorname{cf}[5])$.

- On se donne aussi, $a_{0}: \Omega \times \mathbb{R}^{N} \longrightarrow \mathbb{R}^{N}$ une application de variables $(x, \xi)$ que l'on suppose de Carathéodory et monotone en $\xi$, c'est-à-dire vérifiant

$$
\begin{aligned}
& a_{0}(\cdot, \xi) \text { est mesurable pour tout } \xi \in \mathbb{R}^{N}, a_{0}(x, \cdot) \text { est continue } \\
& \text { sur } \mathbb{R}^{N} \text { p.p. } x \in \Omega
\end{aligned}
$$

$$
\left[a_{0}(x, \xi)-a_{0}(x, \hat{\xi})\right] .(\xi-\hat{\xi}) \geq 0 \quad \text { p.p. } x \in \Omega \text {, pour tout } \xi, \hat{\xi} \in \mathbb{R}^{N}
$$


On suppose de plus qu'il existe des constantes $c, c^{\prime}>0, c_{0} \in L^{1}(\Omega)$ et $c_{1} \in$ $L^{p^{\prime}}(\Omega)$ avec $p^{\prime}$ conjugué de $p$, tel que,

$$
\begin{gathered}
a_{0}(x, \xi) . \xi \geq c|\xi|^{p}-c_{0}(x) \quad \text { p.p. } x \in \Omega, \text { pour tout } \xi \in \mathbb{R}^{N}, \\
\left|a_{0}(x, \xi)\right| \leq c_{1}(x)+c^{\prime}|\xi|^{p-1} \quad \text { p.p. } x \in \Omega, \text { pour tout } \xi \in \mathbb{R}^{N}
\end{gathered}
$$

où $|\cdot|$ est la norme euclidienne de $\mathbb{R}^{N}$.

On définit alors pour tout $u, v \in V$,

$$
a(u, v)=\int_{\Omega} a_{0}(x, D u(x)) \cdot D v(x) d x
$$

$a$ vérifie les hypothèses $(1.2),(1.3)$ et (1.4) grâce à $(5.5),(5.6)$ et (5.8), et $(a, \Phi)$ vérifie l'hypothèse de coercivité (1.6) grâce à la positivité de $\Phi$ et (5.7). Ainsi $(V, a, \Phi)$ vérifie les hypothèses (1.1) à (1.6).

Nous noterons $S(t)=S_{a, \Phi}(t)$ le semi-groupe associé à $(V, a, \Phi)$ défini par (1.8).

Remarque 5.1. i) $S(t)$ est croissant sur $H$. Cela provient de la caractérisation donnée au corollaire 2.2, en remarquant que l'on a,

$$
\begin{gathered}
\Phi(u \wedge \hat{u})+\Phi(u \vee \hat{u})=\Phi(u)+\Phi(\hat{u}), \\
a\left(u,(u-\hat{u})^{+}\right) \geq a\left(\hat{u},(u-\hat{u})^{+}\right) .
\end{gathered}
$$

En effet (5.10) est due à

$$
\begin{aligned}
\varphi(x, \widetilde{u \wedge \hat{u}}(x))+\varphi(x, \widetilde{u \vee \hat{u}}(x)) & =\varphi(x, \tilde{u}(x) \wedge \tilde{\hat{u}}(x))+\varphi(x, \tilde{u}(x) \vee \tilde{\hat{u}}(x)) \\
& =\varphi(x, \tilde{u}(x))+\varphi(x, \tilde{\hat{u}}(x)) \quad \mu p . p . x \in \bar{\Omega}
\end{aligned}
$$

et (5.11) traduit la T-monotonie de $a$ due à (5.6) et

$$
\int_{\Omega} a_{0}(\cdot, D u) \cdot D\left((u-\hat{u})^{+}\right) d x=\int_{u>\hat{u}} a_{0}(\cdot, D u) \cdot(D u-D \hat{u}) d x
$$

ii) $a_{0}(\cdot, 0)=0$ est une condition suffisante pour que $S(t)$ soit positif. En effet, il suffit d'utiliser la caractérisation donnée dans l'exemple 1.3 et (5.3).

Enfin l'on se donne deux autres applications,

$\psi: \bar{\Omega} \times \mathbb{R} \rightarrow[0, \infty]$ vérifiant $(5.1),(5.2),(5.3)$

$b_{0}: \Omega \times \mathbb{R}^{N} \rightarrow \mathbb{R}^{N}$ vérifiant (5.5), (5.6), (5.7), (5.8).

On note pour $u, v \in V$,

$$
\Psi(u)=\int_{\bar{\Omega}} \psi(x, \tilde{u}(x)) d \mu(x), \quad b(u, v)=\int_{\Omega} b_{0}(x, D u(x)) \cdot D v(x) d x .
$$

$T(t)=S_{b, \Psi}(t)$ est le semi-groupe associé à $(V, b, \Psi)$.

\section{Rappels et Remarques 5.2.}

Rappelons (cf. Remarque 2.3, Théorème 2.1 et Remarque 5.1) que, $S(t) \leq$ $T(t)$ pour tout $t \geq 0$ si et seulement si pour tout $v, w \in V$

$$
\begin{aligned}
\int_{\bar{\Omega}} \varphi(\cdot, \tilde{v} \wedge \tilde{w}) d \mu & +\int_{\bar{\Omega}} \psi(\cdot, \tilde{v} \vee \tilde{w}) d \mu \leq \int_{\bar{\Omega}} \varphi(\cdot, \tilde{v}) d \mu+\int_{\bar{\Omega}} \psi(\cdot, \tilde{w}) d \mu \\
& +\int_{v>w}\left(a_{0}(\cdot, D v)-b_{0}(\cdot, D w)\right) \cdot(D v-D w) d x
\end{aligned}
$$


Il est clair que (5.12) est vérifiée si $a_{0}=b_{0}$ et $\partial \psi \leq \partial \varphi$ au sens suivant (cf. [4]),

$$
\partial \psi^{0}(x, h) \leq \partial \varphi^{0}(x, h)^{4} \quad \mu \text { p.p. } x \in \bar{\Omega}, \forall h \in \mathbb{R} .
$$

en notant (cf. [4] preuve du lemme 2.1) que (5.13) est équivalent à

$$
\varphi(x, k)+\psi(x, h) \leq \varphi(x, h)+\psi(x, k) \quad \mu \text { p.p. } x \in \bar{\Omega}, \forall k \leq h .
$$

De même, rappelons (cf. définition 3.1, théorème 3.3, et remarque 5.1) que $S(t)$ est dominé par $T(t)$ si et seulement si $T(t) \geq 0$ pour tout $t \geq 0$ et, pour tout $v \in V$ et tout $w \in V^{+}$on a,

$$
\begin{aligned}
\int_{\bar{\Omega}} \varphi(\cdot,(|\tilde{v}| \wedge \tilde{w}) \operatorname{sign} \tilde{v}) d \mu+\int_{\bar{\Omega}} \psi(\cdot,|\tilde{v}| \vee \tilde{w}) d \mu \\
\leq \int_{\bar{\Omega}} \varphi(\cdot, \tilde{v}) d \mu+\int_{\bar{\Omega}} \psi(\cdot, \tilde{w}) d \mu \\
\quad+\int_{|v|>w}\left(\operatorname{sign} v a_{0}(\cdot, D v)-b_{0}(\cdot, D w)\right) \cdot(D|v|-D w) d x .
\end{aligned}
$$

Il est clair que (5.15) est vérifiée si $a_{0}=b_{0}$ avec $a_{0}(x, \cdot)$ impaire pour p.p. $x \in$ $\Omega$ et $\partial \psi(|h|) \leq|\partial \varphi(h)|$ au sens suivant,

$$
\partial \psi^{0}(x,|h|) \leq\left|\partial \varphi^{0}(x, h)\right| \quad \text { p.p.p. } x \in \bar{\Omega}, \forall h \in \mathbb{R}
$$

en notant que (5.16) est équivalent à,

$$
\begin{aligned}
\varphi(x, k \operatorname{sign} h)+\psi(x,|h|) \leq \varphi(x, h)+\psi(x, k) & \\
& \quad \text { r.p.p. } x \in \bar{\Omega}, \forall 0 \leq k \leq|h| .
\end{aligned}
$$

Nous venons de donner des conditions suffisantes naturelles et pratiquement immédiates de comparaison et de domination. Mais nous ne savons pas donner des conditions nécessaires et suffisantes. Nous pouvons cependant sous certaines hypothèses techniques donner des conditions nécessaires.

Proposition 5.3. Outre les hypothèses faites sur $\varphi, \psi, a_{0}, b_{0}$, l'on suppose de plus que

$$
\begin{aligned}
& \varphi(\cdot, h), \psi(\cdot, h) \in L^{1}(\bar{\Omega}, \mu) \quad \text { pour tout } h \in \mathbb{R}
\end{aligned}
$$

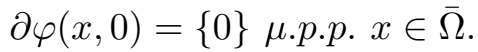

i) Si $S(t) \leq T(t)$ pour tout $t \geq 0$ alors $\partial \psi \leq \partial \varphi$;

ii) Si $S(t)$ est dominé par $T(t)$ alors $\partial \psi(|h|) \leq|\partial \varphi(h)|$.

Proposition 5.4. On suppose $a_{0}-b_{0}$ continue sur $(\Omega \backslash \operatorname{supp} \mu) \times \mathbb{R}^{N}$

\footnotetext{
${ }^{4}$ Pour $\mu$ p.p. $x \in \bar{\Omega}$,

$$
\partial \varphi^{0}(x, .): h \in \mathbb{R} \rightarrow\left\{\begin{array}{l}
\text { inf } \partial \varphi(x, h) \in[0,+\infty] \text { si } h>0, \\
0 \text { si } h=0, \\
\text { sup } \partial \varphi(x, h) \in[0,+\infty] \text { si } h<0
\end{array}\right.
$$

avec la convention inf $\emptyset=+\infty$, sup $\emptyset=-\infty$ et pour $h \in \mathbb{R}, \ell \in \partial \varphi(x, h) \Leftrightarrow \ell \in \mathbb{R}$ et $\ell(\hat{h}-h)+\varphi(x, h) \leq \varphi(x, \hat{h}), \forall \hat{h} \in \mathbb{R}$.
} 
1) Si $S(t) \leq T(t)$ pour tout $t \geq 0 \quad$ (resp. $S(t)$ est dominé par $T(t))$ alors $a_{0}(x, \cdot)-b_{0}(x, \cdot)$ est affine antisymétrique pour tout $x \in \Omega \backslash$ supp $\mu$ et $\operatorname{Div}_{x} a_{0}(\cdot, \xi) \leq \operatorname{Div}_{x} b_{0}(\cdot, \xi)$ dans $\mathcal{D}^{\prime}(\Omega \backslash \operatorname{supp} \mu)$ pour tout $\xi \in \mathbb{R}^{N}$;

2) On suppose de plus $(x, \xi) \rightarrow a_{0}(x, \xi)+a_{0}(x,-\xi)$ continue $\operatorname{sur}(\Omega \backslash$ supp $\mu)$ $\times \mathbb{R}^{N}$, si $S(t)$ est dominé par $T(t)$ alors $a_{0}(x, \cdot)-a_{0}(x, 0)$ et $b_{0}(x, \cdot)-$ $b_{0}(x, 0)$ sont impaires pour tout $x \in \Omega \backslash$ supp $\mu$.

Corollaire 5.5. On suppose que,

- la mesure de Lebesque de supp $\mu$ est nulle,

- $\varphi(\cdot, h), \psi(\cdot, h) \in L^{1}(\bar{\Omega}, \mu)$ pour tout $h \in \mathbb{R}$ et $\partial \varphi(x, 0)=\{0\} \mu . p . p . x \in \bar{\Omega}$

- $a_{0}$ et $b_{0}$ sont continues sur $\Omega \times \mathbb{R}^{N}, a_{0}(x, \cdot)-b_{0}(x, \cdot)$ est un gradient p.p. $x \in \Omega$ et $a_{0}(\cdot, 0)=b_{0}(\cdot, 0)=0$.

Alors

1) $S(t) \leq T(t)$ pour tout $t \geq 0 \Leftrightarrow a_{0}=b_{0}$ et $\partial \psi \leq \partial \varphi$;

2) $S(t)$ est dominé par $T(t) \Leftrightarrow a_{0}=b_{0}, a_{0}(x, \cdot)$ est impaire pour tout $x \in \Omega$ et

$$
\partial \psi|h| \leq|\partial \varphi(h)| .
$$

Preuve de la proposition 5.3. Remarquons tout d'abord que (5.18) implique

$$
\varphi(\cdot, h)<+\infty, \quad \psi(\cdot, h)<+\infty, \quad \text { н.p.p. } x \in \bar{\Omega}, \forall h \in \mathbb{R} .
$$

Montrons $i$ ). On suppose donc (5.12) que l'on utilise avec $w=u \in W^{1, p} \cap$ $\mathcal{C}(\bar{\Omega})$ et $v=u+k$ avec $k$ constante $>0$, on obtient

$$
\int_{\bar{\Omega}} \varphi(\cdot, u) d \mu+\int_{\bar{\Omega}} \psi(\cdot, u+k) d \mu \leq \int_{\bar{\Omega}} \varphi(\cdot, u+k) d \mu+\int_{\bar{\Omega}} \psi(\cdot, u) d \mu<\infty
$$

$W^{1, p}(\Omega) \cap \mathcal{C}(\bar{\Omega})$ étant dense dans $L^{1}(\bar{\Omega}, \mu),(5.21)$ est encore vraie pour $u=$ $h \chi_{A}$ avec $h$ une constante et $A \subset \bar{\Omega} \mu$-mesurable; on a donc

$$
\begin{aligned}
\int_{A} & \frac{\psi(\cdot, h+k)-\psi(\cdot, h)}{k} d \mu+\int_{A^{c}} \frac{\psi(\cdot, k)}{k} d \mu \\
& \leq \int_{A} \frac{\varphi(\cdot, h+k)-\varphi(\cdot, h)}{k} d \mu+\int_{A^{c}} \frac{\varphi(\cdot, k)}{k} d \mu
\end{aligned}
$$

$\operatorname{par}(5.20)$ on a

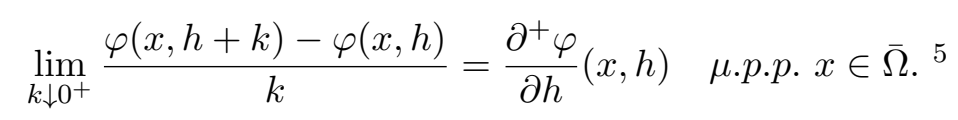

Utilisant la positivité de $\psi, \varphi(\cdot, 0)=0$ et $(5.19)^{6}$ on obtient après passage à la limite lorsque $k \downarrow 0^{+}$dans (5.22),

$$
\int_{A} \frac{\partial^{+} \psi}{\partial h}(\cdot, h) \leq \int_{A} \frac{\partial^{+} \varphi}{\partial h}(\cdot, h) \quad \text { pour tout } A \mu \text {-mesurable et tout } h \in \mathbb{R}
$$

d'où

$$
\frac{\partial^{+} \psi}{\partial h}(x, h) \leq \frac{\partial^{+} \varphi}{\partial h}(x, h) \quad \text { u.p.p. } x \in \bar{\Omega} \forall h \in \mathbb{R} .
$$

$5 \frac{\partial^{+} \varphi}{\partial h}$ est la dérivée à droite par rapport à $h$ de $\varphi$; on a $\frac{\partial^{+} \varphi}{\partial h}(x, h)=\max \partial \varphi(x, h)$.

${ }^{6}$ ici il suffirait d'avoir $\max \partial \varphi(x, 0)=0 \mu$ p.p. $x \in \bar{\Omega}$. 
d'après $(5.20),(5.23)$ est équivalente à la croissance de $\varphi(x, \cdot)-\psi(x, \cdot) \mu . p . p x$ $\in \bar{\Omega}$ donc équivalente à (5.14) et à (5.13).

Montrons ii). On suppose donc (5.15) et l'on procède comme dans la preuve de i) en prenant $w=u \in W^{1, p} \cap \mathcal{C}(\bar{\Omega})^{+}, v=\varepsilon(u+k)$ avec $k$ constante $>0$ et $\varepsilon= \pm 1$ puis $u=h \chi_{A}$ avec $h$ constante $\geq 0$ et $A \subset \bar{\Omega} \mu$-mesurable. On obtient

$$
\begin{gathered}
\qquad \frac{\partial^{+} \psi}{\partial h}(x, h) \leq \frac{\partial^{+} \varphi}{\partial h}(x, h) \quad \mu . p . p . x \in \bar{\Omega} \forall h \in \mathbb{R}^{+} \\
\partial \psi^{0}(x,|h|) \leq \frac{\partial^{+} \psi}{\partial h}(x, h) \leq-\frac{\partial^{-} \varphi}{\partial h}(x, h)=\left|\partial \varphi^{0}(x, h)\right| \mu \cdot p . p . x \in \bar{\Omega}, \forall h \in \mathbb{R}^{-}, \\
\text {sous l'hypothèse }(5.20) \text {, la première inégalité est équivalente à }
\end{gathered}
$$$$
\partial \psi^{0}(x, h) \leq \partial \varphi^{0}(x, h) \quad \text { н.p.p. } x \in \bar{\Omega}, \forall h \in \mathbb{R}^{+}
$$

donc (5.16) est vérifiée.

Preuve de la proposition 5.4. Pour simplifier l'on note $\Omega^{\prime}=\Omega \backslash$ supp $\mu$ qui est un ouvert de $\Omega$ que l'on suppose non vide, et

$$
f(x, \xi)=a_{0}(x, \xi)-b_{0}(x, \xi) \quad \text { pour }(x, \xi) \in \Omega \times \mathbb{R}^{N} .
$$

On suppose donc que $f$ est continue sur $\Omega^{\prime} \times \mathbb{R}^{N}$.

a) On suppose $S(t) \leq T(t)$ pour tout $t \geq 0$. On utilise (5.12) avec $v, w \in$ $\mathcal{D}\left(\Omega^{\prime}\right)$, on obtient grâce à $(5.3)$,

$$
\int_{v>w}\left(a_{0}(\cdot, D v)-b_{0}(\cdot, D w)\right) \cdot(D v-D w) d x \geq 0
$$

puis l'on prend $v=w+t \zeta$ avec $t>0$ et $\zeta \in \mathcal{D}\left(\Omega^{\prime}\right)^{+}$et l'on fait $t \rightarrow 0^{+}$, il vient,

$$
\int_{\Omega}\left(a_{0}(\cdot, D w)-b_{0}(\cdot, D w)\right) \cdot D \zeta d x \geq 0 .
$$

Cette inégalité reste vraie si l'on prend $w \in \mathcal{C}^{\infty}\left(\mathbb{R}^{N}\right)$; en effet si $\rho \in \mathcal{D}\left(\Omega^{\prime}\right)$ avec $\rho=1$ sur supp $\zeta$ alors $w \rho \in \mathcal{D}\left(\Omega^{\prime}\right)$ et $D(w \rho)=D w$ sur supp $\zeta$. Donc par (5.24) on a

$$
\int_{\mathbb{R}^{N}} f(\cdot, D w) \cdot D \zeta d x \geq 0 \quad \text { pour tout } \zeta \in \mathcal{D}\left(\Omega^{\prime}\right)^{+}, w \in \mathcal{C}^{\infty}\left(\mathbb{R}^{N}\right) .
$$

Alors si pour $\xi \in \mathbb{R}^{N}$ on prend $w(x)=\xi . x$ on obtient

$$
\operatorname{Div}_{x} f(\cdot, \xi) \leq 0 \text { dans } \mathcal{D}^{\prime}\left(\Omega^{\prime}\right) .
$$

Ensuite on fixe $x_{0} \in \Omega^{\prime}, \zeta \in \mathcal{D}\left(\mathbb{R}^{N}\right)^{+}, w \in \mathcal{C}^{\infty}\left(\mathbb{R}^{N}\right)$, et l'on utilise (5.25) avec $\zeta_{n}$ et $w_{n}$ définis par,

$$
\zeta_{n}(x)=\zeta\left(n\left(x-x_{0}\right)\right), \quad w_{n}(x)=\frac{1}{n} w\left(n\left(x-x_{0}\right)\right)
$$

en remarquant que pour $n$ assez grand $\zeta_{n} \in \mathcal{D}\left(\Omega^{\prime}\right)^{+}$; il vient après changement de variables,

$$
\int_{\mathbb{R}^{\mathbb{N}}} f\left(x_{0}+\frac{x}{n}, D w(x)\right) \cdot D \zeta(x) d x \geq 0
$$


puis faisant $n \rightarrow+\infty$ en utilisant la continuité de $f$ sur $\Omega^{\prime} \times \mathbb{R}^{N}$, on obtient

$$
\int_{\mathbb{R}^{\mathbb{N}}} f\left(x_{0}, D w(x)\right) \cdot D \zeta(x) d x \geq 0 .
$$

Supposons $\xi \rightarrow f\left(x_{0}, \xi\right)$ de classe $\mathcal{C}^{1}$; il vient en intégrant (5.27) par parties,

$$
\sum_{i, j} \int_{\mathbb{R}^{N}} \frac{\partial f_{i}}{\partial \xi_{j}}\left(x_{0}, D w(x)\right) \frac{\partial^{2} w}{\partial x_{j} \partial x_{i}}(x) \zeta(x) \leq 0 \quad \text { pour tout } \zeta \in \mathcal{D}\left(\mathbb{R}^{N}\right)^{+}
$$

où $f_{1}, \ldots, f_{n}$ sont les composantes de $f$; d'où,

$\sum_{i, j} \frac{\partial f_{i}}{\partial \xi_{j}}\left(x_{0}, D w(x)\right) \frac{\partial^{2} w}{\partial x_{i} \partial x_{j}}(x) \leq 0 \quad$ pour tout $x \in \mathbb{R}^{N}$ et tout $w \in \mathcal{C}^{\infty}\left(\mathbb{R}^{N}\right)$ prenons $w(x)=\xi_{0} \cdot x+\frac{\lambda}{2}(\xi \cdot x)^{2}$ où $\xi_{0}, \xi \in \mathbb{R}^{N}$ et $\lambda \in \mathbb{R}$, puis $x=0$ il vient,

$$
\sum_{i, j} \frac{\partial f_{i}}{\partial \xi_{j}}\left(x_{0}, \xi_{0}\right) \xi_{i} \xi_{j}=0 \text { pour tout } \xi \in \mathbb{R}^{N}
$$

d'où

$$
\frac{\partial f_{i}}{\partial \xi_{j}}\left(x_{0}, \xi_{0}\right)+\frac{\partial f_{j}}{\partial \xi_{i}}\left(x_{0}, \xi_{0}\right)=0 \quad \text { pour tout } i, j \in\{1, . . \cdot, N\} \text { et tout } \xi_{0} \in \mathbb{R}^{N}
$$

Si $\xi \rightarrow f\left(x_{0}, \xi\right)$ n'est pas de classe $\mathcal{C}^{1}$, par une méthode classique de régularisation on obtient,

$$
\frac{\partial f_{i}}{\partial \xi_{j}}\left(x_{0}, \cdot\right)+\frac{\partial f_{j}}{\partial \xi_{i}}\left(x_{0}, \cdot\right)=0 \text { dans } \mathcal{D}^{\prime}\left(\mathbb{R}^{N}\right),
$$

pour tout $i, j \in\{1, . \cdot, N\}$ et tout $x_{0} \in \Omega^{\prime}$. Les solutions de (5.28) sont classiques et données par,

$$
f_{i}\left(x_{0}, \xi\right)=f_{i}^{0}\left(x_{0}\right)+\sum_{j \neq i} f_{i}^{j}\left(x_{0}\right) \xi_{j} \text { pour tout } i \in\{1, . . \cdot, N\} \text { et tout } \xi \in \mathbb{R}^{N}
$$

avec $f_{i}^{j}\left(x_{0}\right)+f_{j}^{i}\left(x_{0}\right)=0$

Cela prouve que $f\left(x_{0}, \cdot\right)$ est affine antisymétrique pour tout $x_{0} \in \Omega^{\prime}$.

b) On suppose $S(t)$ dominé par $T(t)$. On utilise (5.15) avec $w \in \mathcal{D}\left(\Omega^{\prime}\right)^{+}, v=$ $\varepsilon(w+t \zeta)$ où $\varepsilon= \pm 1, t>0$ et $\zeta \in \mathcal{D}\left(\Omega^{\prime}\right)^{+}$on obtient grâce à $(5.3)$

$$
\int_{\Omega}\left(\varepsilon a_{0}(\cdot, \varepsilon D w+t \varepsilon D \zeta)-b_{0}(., D w)\right) \cdot D \zeta \geq 0
$$

et l'on fait $t \rightarrow 0^{+}$pour obtenir

$$
\int_{\Omega}\left(\varepsilon a_{0}(\cdot, \varepsilon D w)-b_{0}(\cdot, D w)\right) \cdot D \zeta \geq 0 .
$$

Cette inégalité reste vraie si l'on prend $w \in \mathcal{C}^{\infty}\left(\mathbb{R}^{N}\right)$; en effet si $\rho \in \mathcal{D}\left(\Omega^{\prime}\right)$ avec $\rho=1$ sur supp $\zeta$ et si $m=$ inf $\rho w$, alors $\rho(w \rho-m) \in \mathcal{D}\left(\Omega^{\prime}\right)^{+}$et $D(\rho(w \rho-m))=D w$ sur supp $\zeta$, d'où

$$
\int_{\mathbb{R}^{N}}\left(\varepsilon a_{0}(\cdot, \varepsilon D w)-b_{0}(\cdot, D w)\right) \cdot D \zeta \geq 0
$$


pour tout $\zeta \in \mathcal{D}\left(\Omega^{\prime}\right)^{+}$et tout $w \in \mathcal{C}^{\infty}\left(\mathbb{R}^{N}\right)$.

En prenant $\varepsilon=1$ on obtient $(5.25)$ et $(5.26)$, et donc $f(x, \cdot)$ est affine antisymétrique pour tout $x \in \Omega^{\prime}$.

En prenant $\varepsilon=-1$ on obtient en posant $g(x, \xi)=-a_{0}(x,-\xi)-b_{0}(x, \xi)$

$$
\int_{\mathbb{R}^{N}} g(\cdot, D w) \cdot D \zeta \geq 0 \quad \text { pour tout } \zeta \in \mathcal{D}\left(\Omega^{\prime}\right)^{+}, w \in \mathcal{C}^{\infty}\left(\mathbb{R}^{N}\right) .
$$

Si l'on suppose de plus que $(x, \xi) \rightarrow a_{0}(x, \xi)+a_{0}(x,-\xi)$ est continue sur $\Omega^{\prime} \times \mathbb{R}^{N}$ alors $g$ est continue sur $\Omega^{\prime} \times \mathbb{R}^{N}$ et l'on déduit comme pour $f$, que $g$ est affine antisymétrique.

Un argument simple de parité montre alors que

$$
f(x, \cdot)-f(x, 0)=g(x, \cdot)-g(x, 0) \text { pour } x \in \Omega^{\prime}
$$

d'où l'on déduit que $a_{0}(x, \cdot)-a_{0}(x, 0)$ et $b_{0}(x, \cdot)-b_{0}(x, 0)$ sont impairs pour tout $x \in \Omega^{\prime}$.

Preuve du corollaire 5.5. C'est une conséquence facile des propositions 5.3 et 5.4 et des remarques 5.1 et 5.2 en remarquant que si $a_{0}(x, \cdot)-b_{0}(x, \cdot)$ est linéaire et est un gradient alors elle est symétrique.

\section{REFERENCES}

[1] H. Attouch and C. Picard, Problèmes variationnels et théorie du potentiel non linéaire, (French) Ann. Fac. Sci. Toulouse Math. (5) 1 (1979), 89-136.

[2] H. Brézis, Oprateurs Maximaux Monotones et Semi-groupes de Contractions dans les Espaces de Hilbert, (French) North-Holland Mathematics Studies, No. 5. Notas de Matemtica (50). North-Holland Publishing Co., Amsterdam-London; American Elsevier Publishing Co., Inc., New York, 1973.

[3] H. Brézis, Équations et inéquations non linéaires dans les espaces vectoriels en dualité, (French) Ann. Inst. Fourier (Grenoble) 18 (1968), 115-175.

[4] P. Bénilan and P. Wittbold, Absorptions non linéaires, (French) J. Funct. Anal. 114 (1993), 59-96.

[5] C. Castaing and M. Valadier, M. Convex Analysis and Measurable Multifunctions, Lecture Notes in Math., \#580, Springer-Verlag, Berlin-New York, 1977.

[6] T. Kato, Perturbation Theory of Linear Operators, Springer-Verlag, Berlin, 1966.

[7] E. M. Ouhabaz, $L^{\infty}$-contractivity of semigroups generated by sectorial forms, J. London Math. Soc. (2) 46 (1992), 529-542.

[8] E. M. Ouhabaz, Invariance of closed convex sets and domination criteria for semigroups, Potential Analysis, Kluwer Acad. Publ., 1996, to appear.

[9] R. R. Phelps, Convex Functions, Monotone Operators and Differentiability, Lecture Notes in Math., \#1364, Springer-Verlag, New York, 1989.

Equipe de MathÉMatiques

U.R.A. CNRS 741

Université de Franche-Comté

25030 Besançon Cedex, FRANCE

E-mail address: barthelemy@math.univ-fcomte.fr 


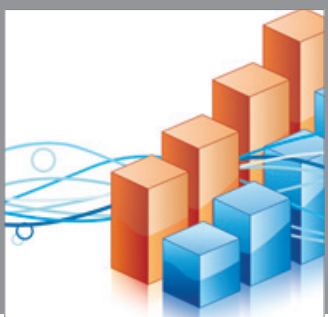

Advances in

Operations Research

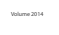

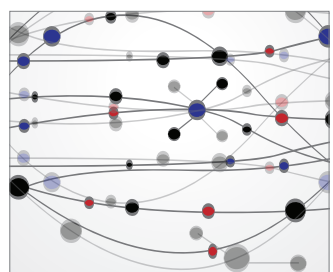

\section{The Scientific} World Journal
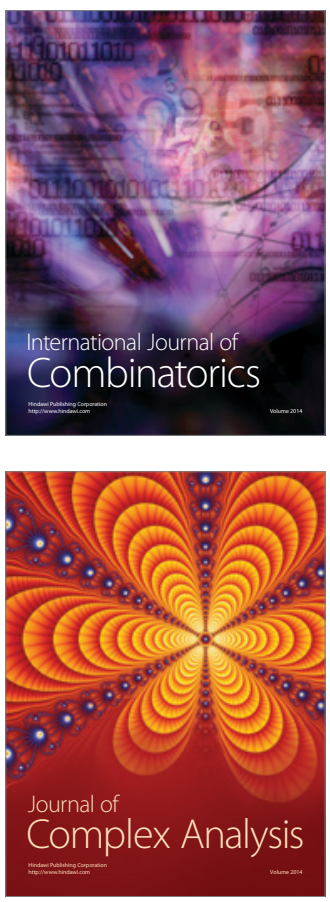

International Journal of

Mathematics and

Mathematical

Sciences
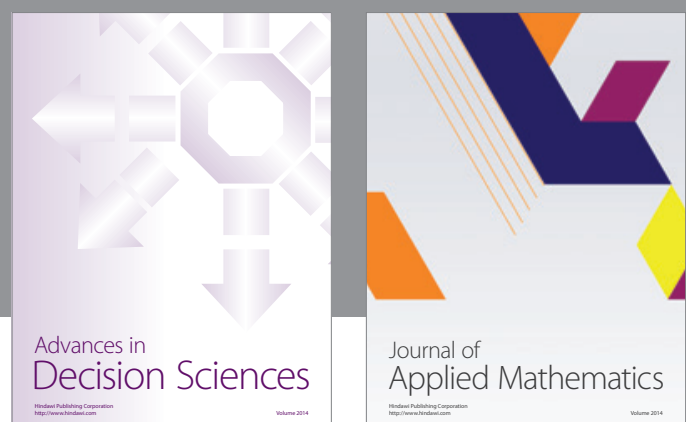

Journal of

Applied Mathematics
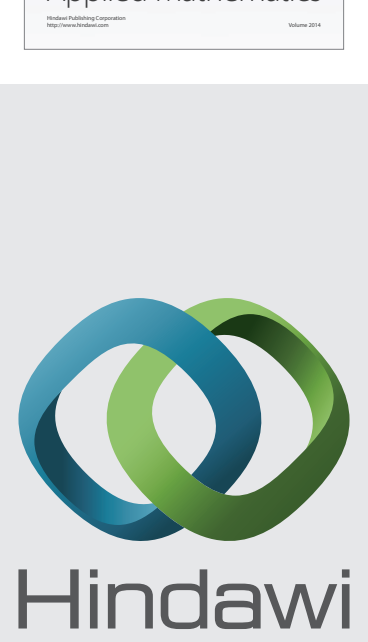

Submit your manuscripts at http://www.hindawi.com
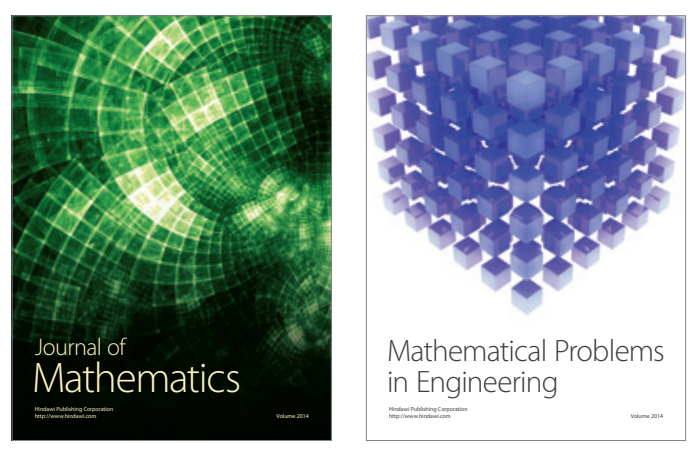

Mathematical Problems in Engineering
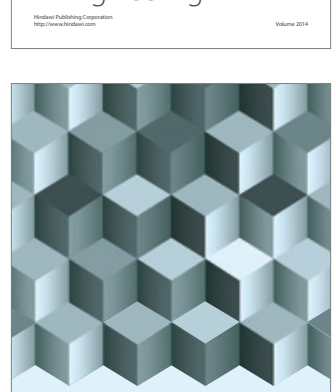

Journal of

Function Spaces
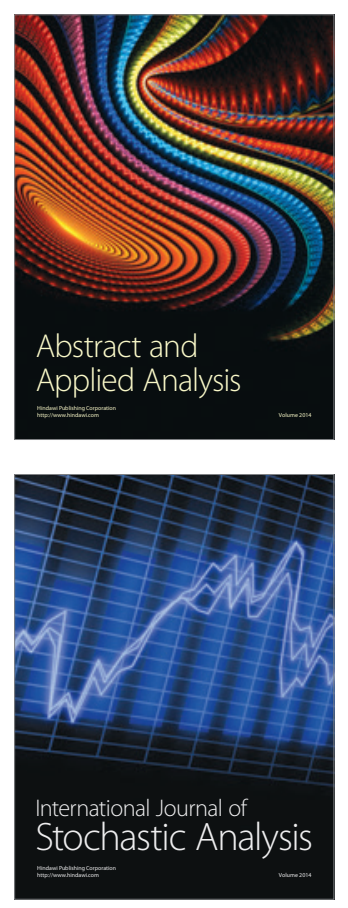

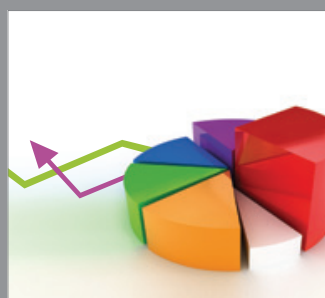

ournal of

Probability and Statistics

Promensencen
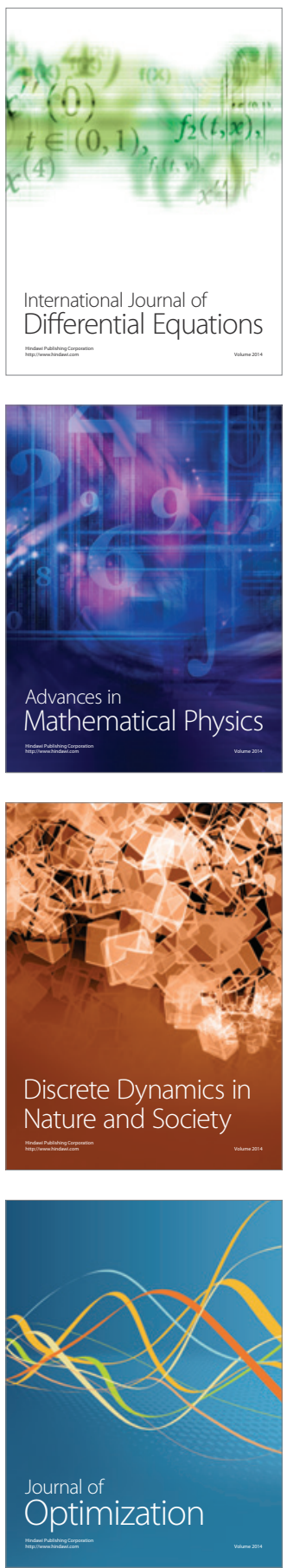